\title{
A competitive solution for cooperative truckload delivery
}

\author{
Behzad Hezarkhani ${ }^{1}$ - Marco Slikker ${ }^{1}$. \\ Tom Van Woensel ${ }^{1}$
}

Received: 16 June 2014 / Accepted: 19 February 2015 / Published online: 18 March 2015

(C) The Author(s) 2015. This article is published with open access at Springerlink.com

\begin{abstract}
This paper introduces a solution for gain sharing in consortia of logistic providers where joint planning of truckload deliveries enables the reduction of empty kilometers. The highly competitive nature of freight transport markets necessitates solutions that distinguish among the logistics providers based on their characteristics, even in situations with two players only. We introduce desirable properties in these situations and propose a solution that satisfies such properties. By comparing the existing solutions against the introduced properties we demonstrate the advantages of our proposed solution.
\end{abstract}

Keywords Transportation and logistics · Supply chain management . Cooperative game theory $\cdot$ Gain sharing

\section{Introduction}

Road freight constitutes the most dominant form of transportation. However, the industry suffers from significant inefficiencies. In 2012, more than $24 \%$ of all the distance driven by commercial vehicles in European Union was empty (Eurostat 2012). This paper is motivated by a project initiated by two major European logistics providers to create a consortium for cooperative planning of truckload delivery requirements of joining companies to, among others, reduce the costs of empty kilometers. Cruijssen et al. (2007) discuss the other benefits that logistics providers could achieve

$\triangle$ Behzad Hezarkhani

b.hezarkhani@tue.nl

1 School of Industrial Engineering, Eindhoven University of Technology, P.O. Box 513, 5600 MB Eindhoven, The Netherlands 
by cooperation. We address the important issue of sharing the gains obtained from joint planning.

Cooperative truckload delivery (CTLD) situations comprise a number of logistics providers, their resources (e.g., depots, trucks, drivers, equipments, etc.), and their delivery requirements. A delivery requirement can simply be considered as an order for picking up cargo at some location and transporting it to another location. But it may actually involve delivery time windows, special equipments and personnel, and other practical constraints. The delivery requirements must be fulfilled by vehicles in feasible trips. The feasibility of a trip depends on the number and type of deliveries it fulfills, specific depots and equipment that must be employed, and other details.

The optimal delivery plans of individual companies in most cases include a significant amount of unavoidable repositioning movements, i.e., empty kilometers, among their depots and various pickup/delivery locations. By taking advantage of the synergy in aggregated networks of depots and delivery requirements, cooperating companies can decrease their overall empty kilometers. As the cooperating companies are usually in direct competition with each other, it is absolutely critical for them to understand how the cooperation would benefit them as well as their competitors. Thus, the existence of formal models that unambiguously determine allocations of gains and justify their fairness and/or competitiveness in these situations are imperative to success of such consortia.

There are many simple ways to divide the savings among cooperating logistics providers. Such simple ways often divide the savings proportional to some measure defined harmoniously for all players, e.g., number and/or amount of exchanged deliveries, additional costs incurred, empty kilometers avoided, or contributions to total savings. However, despite their practical appeal, simple solutions often fail to produce outcomes which are desirable in terms of fairness/competitiveness in these situations. But what constitutes a desirable allocation in these situations? In this paper, we introduce a set of formal properties that have the ability to capture the notions of fairness and/or competitiveness with regard to allocations in CTLD situations.

The gain-sharing problems in the literature are often approached via the well-known solutions developed in cooperative game theory. By abstracting a cooperative situation into a cooperative game, consisting of a player set and a function that determines the amount of gains attainable by different groups of players, cooperative game theory studies allocations that satisfy collections of logically desirable properties expressed in relation to such an abstraction. In some situations, however, the properties expressible in relation to their associated cooperative games are insufficient to capture all desirable requirements of allocations. Therefore, by disregarding the information contained in the underlying cooperative situation, indispensable properties in some cooperative situations will be impossible to formalize. The cooperative organizations of logistics providers assessed in this paper are instances of such cooperative situations. In this paper, we allow solutions to draw upon the cooperative situations to determine the allocations of savings.

The highly competitive nature of logistics markets as well as the limited number of potential participants necessitate solutions that are capable of incorporating the notion of competitiveness to distinguish among the logistics providers. Such a requirement implores solutions that could potentially distinguish among the logistics providers who 
are identical in terms of their contribution to the obtained savings. A typical example of the latter case is situations with two players only. Most of the well-known solutions in cooperative game theory, e.g., Shapley value and nucleolus, are incapable of differentiating among the allocations in two-player situations. Nevertheless, a number of papers in the OR/OM literature introduce alternative solutions to tackle the latter drawback, e.g., Frisk et al. (2010). However, we show that the available solutions in the literature do not satisfy the properties that are desirable in CTLD situations introduced in this paper.

A part of the desirable properties of solutions in CTLD situations can be expressed in relation to cooperative games associated with those situations. The nonemptiness property demands at least one allocation in every situation. The uniqueness property distinguishes solutions that upon nonemptiness, yield a single allocation in every situation. This property is needed so that no further negotiations would be required to choose among multiple possible allocations. Finally, the least-instability property prescribes allocations that minimize the incentives for groups of players to organize cooperation within themselves. In addition to these rather standard properties, we introduce two new properties which are specific to CTLD situations.

The first desirable property defined specifically for CTLD situations is the independence of irrelevant deliveries property (IID) which states that the allocated savings to the players must be insensitive to parts of their networks which could not have any possible contribution to the savings obtained from cooperation. In this regard, this property defines a boundary for the relevant scope of operation for every logistics provider such that anything beyond this scope should be ignored in allocation of savings. If a solution for CTLD situations does not satisfy this property, then the logistics providers could find it beneficial to inflate their announced delivery requirements in a cooperative organization and small companies with high contribution to the savings could be discriminated against the large companies which may not have significant contributions.

The last property introduced in this paper addresses the ability of solutions to consider the competitive positions of cooperating logistics providers. Although there is no standard measure of competitive positions in logistics markets, we draw upon the notation of average cost of fulfillment to define one. The average cost of fulfillment of a set of delivery requirements is the minimum cost of its fulfillment divided by the amount of full kilometers involved. The motivation for using the average cost of fulfillment as a measure of competitiveness is its pricing implications. Suppose that a company has to announce a fixed price for a unit distance of its delivery services. The average cost of fulfillment then represents the lowest unit price at which a logistics provider neither makes profit nor incurs loss. Thus, if the average cost of fulfillment of the logistics provider $i$ is lower than that of $j, i$ would be able to announce a lower unit price for its delivery services while making a profit. We take this as an indication that prior to cooperation $i$ is in a better competitive position. An allocation of savings to players could alter the average costs of fulfillment after cooperation. Under certain conditions, the restricted competitiveness property requires that solutions equalize the ratio of average costs of fulfillment across players before and after cooperation. In this regard, the appropriate solutions in CTLD situations preserve the competitive positions of logistics providers. 
We propose a solution that satisfies the listed properties in all CTLD situations. In doing so, we first introduce the essential deliveries of a player as subsets of delivery requirements of the player which are necessary and sufficient in bringing about its contribution to cost savings in the grand coalition. When the optimal delivery plan of the grand coalition is unique, the essential deliveries correspond to the deliveries whose fulfillment trips in the grand coalition involve some other players. Therefore, the deliveries which are not essential can be fulfilled independently by their corresponding players as efficiently as in the grand coalition. A preliminary version of our proposed solution equalizes the average cost of fulfillments of essential deliveries of the players before and after cooperation. Although this solution can be easily implemented in such situations, it does not necessarily produce stable outcomes. Our final solution yields a unique point in the core (Gillies 1959), or when the latter is empty, in the least-core (Maschler et al. 1979) which has the shortest distance to the aforementioned allocation.

The rest of this paper is organized as following. In Sect. 2, we discuss the literature on allocation problems with special focus on logistics and transportation context. In Sect. 2, we model a general class of truckload delivery problems and in Sect. 4 we introduce the cooperative version of such situations. The desirable properties in CTLD situations are defined in Sect. 5. In Sect. 6, we develop our proposed solution for CTLD situations. Specifically, Sect. 6.2 outlines the formula for the proposed solution and shows that it satisfies all the listed properties. Section 7 discusses some of the known solutions in the literature in line with the properties introduced in this paper. Section 8 concludes the paper.

\section{Literature review}

There is an extensive literature on allocation problems arising in cooperative operations. Tijs and Driessen (1986) provide a structured view of general cost allocation methods along with references to early adoption of such methods in practice. The literature on cooperative logistics operations, on the other hand, is relatively recentmainly due to the industry's shrinking margins and advances in information technology which motivate and facilitate cooperation.

To deal with allocation problems in logistics and transportation context, many authors have proposed the adoption of well-known solutions of cooperative game theory. Krajewska et al. (2007) discuss the implementation of the Shapley value (Shapley $1953 \mathrm{~b}$ ) as the solution in cooperative organizations of logistics providers. Özener and Ergun (2008) study CTLD situations where all logistics providers have available depots at every location and show that the core (Gillies 1959) of games associated with these situations are always non-empty and dual solutions provide allocations in their core. Hezarkhani et al. (2014) further delineate the possibilities and impossibilities for a complete characterization of the core of these games via dual solutions. In cooperative vehicle routing situations, where the core could be empty or it may include many elements, Göthe-Lundgren et al. (1996) and Engevall et al. (2004) elaborate on the implementation of the nucleolus (Schmeidler 1969) as the solution of choice.

Several papers in the recent literature investigate solutions that incorporate some proportional measures defined on specific features of underlying situations to divide the savings/costs among the logistics providers. Frisk et al. (2010) propose a solution that 
draws upon the stand-alone costs of individual companies. Their suggested solution, i.e., the equal profit method (EPM), chooses allocations in the core, or in the leastcore (Maschler et al. 1979) when the core is empty, such that the spread of ratios of allocated savings to stand-alone costs across all players is minimized. A similar method is proposed independently by Drechsel and Kimms (2010). Multiple extensions of this solution have been proposed ever since. Audy et al. (2010) extend the EPM by including additional constraints that ensure a minimum allocation of savings for all logistics providers. Liu et al. (2010) directly incorporate the marginal contributions of players as weights into the EPM formulation. Dai and Chen (2012) draw attention to allocations in the core with the property that the greatest deviation from the Shapley value is minimum. Finally, Vanovermeire et al. (2013) and Lozano et al. (2013) discuss the different outcomes of various solutions in transportation contexts via numerical examples. What seems to be lacking in this stream of research is the formal definition of situation-specific properties that solutions are expected to satisfy.

Another stream of research investigate the special structures in cooperative games associated with simplified delivery problems. Hamers (1997) analyzes the cooperative Chinese postman games and Hamers et al. (1999) discuss the cost allocation problem in these situations. Granot et al. (1999) investigate the special classes of single-depot delivery games whose cores are always non-empty. Platz and Hamers (2013) characterize the graphs whose induced multi-depot Chinese postman games have non-empty cores. We refer the reader to Curiel (2008) for an overview of cooperative games associated with logistics/transportation situations. Nevertheless, the complexity of games associated with combinatorial situations have given rise to new research frontiers that seek reasonable theoretical compromises in finding good solutions (Caprara and Letchford 2010).

\section{Stand-alone truckload delivery situations}

Stand-alone truckload delivery situations, hereafter STLD situations, reflect the key features of centralized planning in road freight sector. Let $V$ be a set of nodes corresponding to spatial locations and $w: V \times V \rightarrow \mathbb{R}^{+}$be a distance function which satisfies triangular inequalities. A set of $m$ delivery requirements $\boldsymbol{D}=\left\{d^{1}, \ldots, d^{m}\right\}$ is given. A delivery requirement $d^{k} \in \boldsymbol{D}$ is a pair $\left(a^{k}, b^{k}\right) \in V \times V$ consisting of the corresponding pickup location $a^{k}$ and delivery location $b^{k}$. The fulfillment of the delivery requirement $d^{k}$ corresponds to a single traverse of the $\operatorname{arc}\left(a^{k}, b^{k}\right)$ for $k$, i.e., two delivery requirements with identical pickup and delivery locations correspond to two non-identical fulfillments. We assume that the distance between the pickup and delivery locations of every trip is positive. A non-empty set of available depots $\boldsymbol{O}=\left\{o^{1}, \ldots, o^{h}\right\} \subseteq V$ stations vehicles that fulfill the delivery requirements. We assume that the fleet of vehicles is homogeneous and a vehicle has enough capacity to handle every single delivery requirement.

Delivery requirements must be fulfilled in trips. A trip is a sequence of deliveries that starts and ends at a particular depot. The truckload feature of the problem reflects the assumption that for any two delivery requirements, their corresponding cargo cannot be picked up sequentially in a trip without delivering the first-picked cargo. Formally, a trip $l$ is a tuple $\left(o^{l}, D^{l}, \sigma^{l}\right)$ where $o^{l} \in \boldsymbol{O}$ is the origin/destination, $D^{l}$ is 
a subset of deliveries in $\boldsymbol{D}$ that are fulfilled in $l$, and $\sigma^{l}$ is an ordering of deliveries in $D^{l}$ which represents the sequence of fulfillments in trip $l$. Let $\mathcal{L}$ be the set of all such trips. To incorporate the practical constraints that could render some trips infeasiblee.g., delivery time windows, number of possible trips per day, traffic network-we introduce the feasible trip set $L \subseteq \mathcal{L}$. A sub-trip of $l$ is a trip which has the same origin as $l$ and a subset of $l$ 's deliveries whose sequence of fulfillments are consistent with ordering of deliveries in $l$. We assume that if $l$ is within the feasible trip set, its sub-trips are also in the feasible set. Let $L(O, D)=\left\{l \in L \mid o^{l} \in O, D^{l} \subseteq D\right\}$ be the set of all feasible trips that can be used to satisfy deliveries in $D \subseteq \boldsymbol{D}$ from depots in $O \subseteq \boldsymbol{O}, O \neq \emptyset$. Note that $L(\boldsymbol{O}, \boldsymbol{D})=L$. The feasible trip sets are assumed to be rich enough to enable the fulfillment of all delivery requirements. Formally, we assume that for every $d^{k} \in D \subseteq \boldsymbol{D}$ and $O \subseteq \boldsymbol{O}, O \neq \emptyset$, there exists $l \in L(O, D)$ such that $d^{k} \in D^{l}$. Due to homogeneity of the fleet, the feasible trip sets are independent of the choice of vehicle. A truckload delivery situation is characterized by a tuple

$$
\Lambda=(V, w, \boldsymbol{D}, \boldsymbol{O}, L) .
$$

We assume that cost and distance of travel are linearly proportional and without loss of generality normalize the proportion to one. The cost of the feasible trip $l$, $D^{l} \neq \emptyset$, is comprised of two parts. The first part is the cost associated with the distance traveled between the pickup and delivery locations. The full kilometers cost of a trip is independent of both the choice of the trip's depot and the sequence of fulfillments:

$$
c_{F}^{l}=\sum_{k: d^{k} \in D^{l}} w\left(a^{k}, b^{k}\right) .
$$

The second part of a trip's cost, i.e., empty kilometers cost, is the cost associated with the distance traveled from/to the depot and among different fulfillments:

$$
c_{E}^{l}=w\left(o^{l}, a^{\sigma_{1}^{l}}\right)+\sum_{j=1}^{\left|D^{l}\right|-1} w\left(b^{\sigma_{j}^{l}}, a^{\sigma_{j+1}^{l}}\right)+w\left(b^{\sigma^{l} D^{l} \mid}, o^{l}\right)
$$

where the shorthand notation $\sigma_{j}^{l}$ represents the index of the delivery requirement that is fulfilled after all the $j-1$ deliveries preceding it in $\sigma^{l}$ are fulfilled. By $\left|D^{l}\right|$ we denote the number of deliveries in $D^{l}$. The cost of trip $l$ is defined by $c^{l}=c_{F}^{l}+c_{E}^{l}$.

A fulfillment plan $P$, hereafter a plan, from $O$ to $D$ is a collection of feasible trips in $L(O, D)$ that fulfills all deliveries in $D$ exactly once. The assumptions on the richness of feasible trip sets and the feasibility of sub-trips discussed earlier ensure that for every $D \subseteq \boldsymbol{D}$ and every $O \subseteq \boldsymbol{O}, O \neq \varnothing$, a fulfillment plan exists. The deliveries fulfilled in the trips of the plan $P$ partition the corresponding set of delivery requirements, i.e., $\bigcup_{l \in P} D^{l}=D$ and $D^{l} \cap D^{k}=\emptyset$ for all $k, l \in P$ with $l \neq k$. The cost of the plan $P$ is the total cost of its trips, i.e., $c(P)=\sum_{l \in P} c^{l}$. Accordingly, $c(P)$ is decomposable into full and empty parts:

$$
c(P)=c_{F}(P)+c_{E}(P),
$$


where $c_{F}(P)=\sum_{l \in P} c_{F}^{l}$ and $c_{E}(P)=\sum_{l \in P} c_{E}^{l}$ are the total costs of full and empty kilometers of $P$, respectively.

Let $\mathcal{P}(O, D)$ denote the set of all possible fulfillment plans from $O$ to $D$. We call $P \in \mathcal{P}(O, D)$ an optimal plan from $O$ to $D$ if

$$
c(P) \leq c\left(P^{\prime}\right) \text { for all } P^{\prime} \in \mathcal{P}(O, D)
$$

The set of all optimal plans from $O$ to $D$ is denoted by $\mathcal{P}^{*}(O, D)$. If there are multiple optimal plans from $O$ to $D$, their costs are equal. We denote the minimum cost of delivery from $O$ to $D$ with $c^{*}(O, D)$, and the full kilometers cost of $D$, which is independent of the choice of depots, with $c_{F}(D)$. For the purpose of this paper, we hereafter assume that the optimization problems of aforementioned sorts can be readily solved.

We provide some observations which will be used in the rest of the paper.

Lemma 1 Let $\Lambda$ be an STLD situation. Then,

(i) $c^{*}(O, D) \geq c^{*}\left(O, D^{\prime}\right)$ for all $\emptyset \neq O \subseteq \boldsymbol{O}$ and $D^{\prime} \subset D \subseteq \boldsymbol{D}$,

(ii) $c^{*}(O, D) \leq c^{*}\left(O^{\prime}, D\right)$ for all $O^{\prime} \subset O \subseteq \boldsymbol{O}$ and $D \subseteq \boldsymbol{D}$,

(iii) $c^{*}(O, D)+c^{*}\left(O, D^{\prime}\right) \geq c^{*}\left(O, D \cup D^{\prime}\right)$ for all $\emptyset \neq O \subseteq \boldsymbol{O}$ and $D, D^{\prime} \subseteq \boldsymbol{D}$ with $D \cap D^{\prime}=\emptyset$.

Proof (i) Let $D^{\prime} \subset D \subseteq D$ and define $D^{\prime \prime}=D \backslash D^{\prime}$. Let $P \in \mathcal{P}^{*}(O, D)$ be an optimal plan from $O$ to $D$. For every $l \in P$ construct its sub-trip $l^{\prime}$ with $D^{l^{\prime}}=$ $D^{l} \backslash D^{\prime \prime}$. By the assumption on the feasibility of sub-trips, the plan $P^{\prime}$ obtained in this manner is a feasible plan from $O$ to $D^{\prime}$. As the triangular inequalities hold we have $c^{l^{\prime}} \leq c^{l}$ for every $l \in P$. Since the cost of a plan is the total sum of the costs of its trips we have $c\left(P^{\prime}\right) \leq c(P)=c^{*}(O, D)$. Considering that any optimal plan from $O$ to $D^{\prime}$ is at most as costly as $c\left(P^{\prime}\right)$ we have $c^{*}\left(O, D^{\prime}\right) \leq c^{*}(O, D)$.

(ii) Let $O^{\prime} \subset O \subseteq \boldsymbol{O}$ and suppose $P^{\prime} \in \mathcal{P}^{*}\left(O^{\prime}, D\right)$ is an optimal plan from $O^{\prime}$ to $D$. Note that $P^{\prime}$ is a feasible plan from $O$ to $D$ as well since for any trip $l \in P^{\prime}$ we have $o^{l} \in O$. By definition of optimal plans it must be that $c^{*}(O, D) \leq c^{*}\left(O^{\prime}, D\right)$.

(iii) Let $D, D^{\prime} \subseteq D$ be such that $D \cap D^{\prime}=\emptyset$. Let $P \in \mathcal{P}^{*}(O, D)$ and $P^{\prime} \in$ $\mathcal{P}^{*}\left(O, D^{\prime}\right)$. Note that $P \cap P^{\prime}=\varnothing$ and $P \cup P^{\prime}$ is a feasible plan from $O$ to $D \cup D^{\prime}$. Furthermore,

$$
c\left(P \cup P^{\prime}\right)=\sum_{l \in P \cup P^{\prime}} c^{l}=\sum_{l \in P} c^{l}+\sum_{l \in P^{\prime}} c^{l}=c^{*}(O, D)+c^{*}\left(O, D^{\prime}\right) .
$$

By definition of optimal plans we have $c^{*}(O, D)+c^{*}\left(O, D^{\prime}\right) \geq c^{*}\left(O, D \cup D^{\prime}\right)$.

Part (i) of Lemma 1 shows that shrinking the set of delivery requirements cannot increase the minimum cost of delivery. A reverse effect is shown in part (ii) for the depots, that is, augmenting the set of depots cannot increase the minimum cost of delivery. Finally, part (iii) demonstrates the subadditive effect with regard to the minimum costs of fulfillment that results from aggregated planning of delivery requirements. 


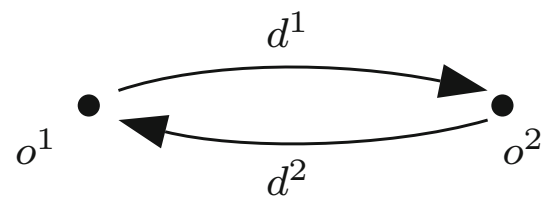

Fig. 1 An STLD situation

We define the average cost of fulfillment from $O$ to $D \neq \emptyset$ as

$$
z(O, D)=\frac{c^{*}(O, D)}{c_{F}(D)}
$$

The average cost of fulfillment $z(O, D)$ represents the cost for fulfilling a unit distance of delivery on average. ${ }^{1}$ If one were supposed to determine a fixed price for every unit distance of delivery services, the average cost of fulfillment would represent the minimum price at which no loss is incurred. Note that the full kilometer cost of a nonempty delivery set is strictly positive by assumption. For $D=\emptyset$, we let $z(O, \emptyset)=0$.

Example 1 Figure 1 depicts an STLD situation with two locations, two depots and two delivery requirements. The distance between the two locations is one and the trip which fulfills both deliveries is feasible. We have $c^{*}(O, D)=2$ and $z(O, D)=1 . \triangle$

The last lemma in this section establishes the connection between the separability of optimal delivery plans and the additivity of their costs.

Lemma 2 Let $\Lambda$ be an STLD situation. Let $\emptyset \neq O^{\prime} \subseteq O \subseteq \boldsymbol{O}$ and $D^{\prime} \subseteq D \subseteq \boldsymbol{D}$. We have $c^{*}\left(O^{\prime}, D^{\prime}\right)+c^{*}\left(O, D \backslash D^{\prime}\right)=c^{*}(O, D)$ if and only iffor every $P^{\prime} \in \mathcal{P}^{*}\left(O^{\prime}, D^{\prime}\right)$ and $P \in \mathcal{P}^{*}\left(O, D \backslash D^{\prime}\right)$, it holds that $P \cup P^{\prime} \in \mathcal{P}^{*}(O, D)$.

Proof (If) Let $P^{\prime} \in \mathcal{P}^{*}\left(O^{\prime}, D^{\prime}\right)$ and $P \in \mathcal{P}^{*}\left(O, D \backslash D^{\prime}\right)$ and assume that $P \cup P^{\prime} \in$ $\mathcal{P}^{*}(O, D)$. By definition of minimum cost of delivery we have

$$
c^{*}(O, D)=\sum_{l \in P^{\prime} \cup P} c^{l}=\sum_{l \in P^{\prime}} c^{l}+\sum_{l \in P} c^{l}=c^{*}\left(O^{\prime}, D^{\prime}\right)+c^{*}\left(O, D \backslash D^{\prime}\right) .
$$

(Only if) Assume that $c^{*}\left(O^{\prime}, D^{\prime}\right)+c^{*}\left(O, D \backslash D^{\prime}\right)=c^{*}(O, D)$. Let $P^{\prime} \in \mathcal{P}\left(O^{\prime}, D^{\prime}\right)$ and $P \in \mathcal{P}\left(O, D \backslash D^{\prime}\right)$. Note that $P \cup P^{\prime}$ is a feasible delivery plan from $O$ to $D$. We have

$$
c\left(P^{\prime} \cup P\right)=\sum_{l \in P^{\prime} \cup P} c^{l}=c^{*}\left(O^{\prime}, D^{\prime}\right)+c^{*}\left(O, D \backslash D^{\prime}\right)=c^{*}(O, D)
$$

where the last equality follows by assumption. Hence, $P^{\prime} \cup P \in \mathcal{P}^{*}(O, D)$.

\footnotetext{
${ }^{1}$ The equivalence of cost and distance of travel assumed in this paper is for simplifying the notation. When this assumption is relaxed, the denominator in Eq. (5) must be replaced with the total distance of full kilometers in $D$.
} 


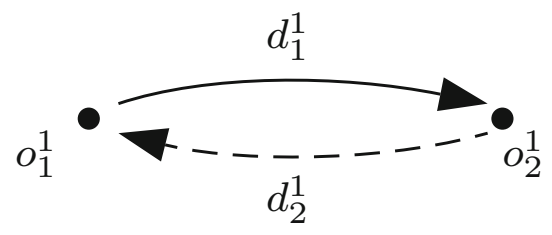

Fig. 2 A CTLD situation

\section{CTLD situations and games}

This section introduces the cooperative versions of truckload delivery situations wherein a number of logistics service providers, hereafter players, have the option to jointly optimize their fulfillment plans. While these situations reflect the key features of decentralized road freight markets, their associated cooperative games formalize the underlying gain-sharing problems.

CTLD situations are extensions of STLD situations. Let $\Lambda=(V, w, \boldsymbol{D}, \boldsymbol{O}, L)$ be an STLD situation. Consider a non-empty set $N=\{1, \ldots, n\}$ of players. Each player $i \in N$ possesses a set of delivery requirements $D_{i} \subseteq \boldsymbol{D}$ and a non-empty set of depots $O_{i} \subseteq \boldsymbol{O}$ such that $\cup_{i \in N} D_{i}=\boldsymbol{D}$ and $\cup_{i \in N} O_{i}=\boldsymbol{O}$. The fleets of players' vehicles are homogeneous and capacious enough to handle the cargo corresponding to every single delivery requirement.

A coalition is a subset of players. Let $O_{S}=\cup_{i \in S} O_{i}$ and $D_{S}=\cup_{i \in S} D_{i}$ denote the combined set of depots and delivery requirements of players in coalition $S \subseteq N$. The set $L\left(O_{S}, D_{S}\right)$ contains all feasible trips that coalition $S \subseteq N$ can use to fulfill its combined delivery requirements. Note that $L\left(O_{N}, D_{N}\right)=L$. By the assumption on the richness of feasible trip sets, any coalition of players is able to fulfill its deliveries. A CTLD situation is a tuple:

$$
\Gamma=\left(N, V, w,\left(D_{i}\right)_{i \in N},\left(O_{i}\right)_{i \in N}, L\right)
$$

with all elements being as described previously. We denote the set of all possible CTLD situations with $\mathcal{T}$.

By joint planning of fulfillments, a coalition in a CTLD situation could reduce the cost of its empty kilometers and thus obtain savings. The savings generated by a coalition can be due to utilization of a larger pool of depots for constructing trips or combining fulfillments together more efficiently in trips, or both.

The cost savings obtained by a coalition $S \subseteq N$ in $\Gamma$ is

$$
\sum_{i \in S} c^{*}\left(O_{i}, D_{i}\right)-c^{*}\left(O_{S}, D_{S}\right) .
$$

Recall that $c^{*}\left(O_{S}, D_{S}\right)$ is the cost of an optimal plan from $O_{S}$ to $D_{S}$ in the STLD situation corresponding to $\Gamma$.

Example 2 Figure 2 depicts a CTLD situation $\Gamma$ with two players, $N=\{1,2\}$, each having a single depot $o_{1}^{1}$ and $o_{2}^{1}$, and a single delivery requirement $d_{1}^{1}$ and $d_{2}^{1}$, respectively. The pickup location of each player's delivery requirement is its depot and the 
delivery location coincides with the other player's depot. Assuming that the distance between the two locations is one kilometer, we have $c^{*}\left(O_{1}, D_{1}\right)=c^{*}\left(O_{2}, D_{2}\right)=$ $c^{*}\left(O_{N}, D_{N}\right)=2$. The cost saving obtained by the grand coalition is 2 .

The cooperative CTLD game associated with the situation $\Gamma \in \mathcal{T}$ with player set $N$ is the pair $\left(N, v^{\Gamma}\right)$ where for every $S \subseteq N$ :

$$
v^{\Gamma}(S)=\sum_{i \in S} c^{*}\left(O_{i}, D_{i}\right)-c^{*}\left(O_{S}, D_{S}\right) .
$$

The cooperative game $\left(N, v^{\Gamma}\right)$ is superadditive if for all $S, T \subseteq N$ such that $S \cap T=\varnothing$ it holds that $v^{\Gamma}(S \cup T) \geq v^{\Gamma}(S)+v^{\Gamma}(T)$. If the game is superadditive, then the savings obtained in the grand coalition $N$ is never less than the total savings obtained by any other partitioning of players into coalitions. The following theorem states that games associated with CTLD situations are generally superadditive.

Theorem 1 For every CTLD situation $\Gamma \in \mathcal{T}$ with player set $N$, the associated cooperative game $\left(N, v^{\Gamma}\right)$ is superadditive.

Proof Let $\Gamma \in \mathcal{T}$ be a CTLD situation with player set $N$. Let $S, T \subseteq N$ such that $S \cap T=\emptyset$. Then,

$$
\begin{aligned}
v^{\Gamma}(S \cup T) & =\sum_{i \in S \cup T} c^{*}\left(O_{i}, D_{i}\right)-c^{*}\left(O_{S \cup T}, D_{S \cup T}\right) \\
& \geq \sum_{i \in S \cup T} c^{*}\left(O_{i}, D_{i}\right)-c^{*}\left(O_{S \cup T}, D_{S}\right)-c^{*}\left(O_{S \cup T}, D_{T}\right) \\
& \geq \sum_{i \in S \cup T} c^{*}\left(O_{i}, D_{i}\right)-c^{*}\left(O_{S}, D_{S}\right)-c\left(O_{T}, D_{T}\right) \\
& =\sum_{i \in S} c^{*}\left(O_{i}, D_{i}\right)-c^{*}\left(O_{S}, D_{S}\right)+\sum_{i \in T} c^{*}\left(O_{i}, D_{i}\right)-c\left(O_{T}, D_{T}\right) \\
& =v^{\Gamma}(S)+v^{\Gamma}(T)
\end{aligned}
$$

The first inequality follows from part (iii) of Lemma 1 and the second inequality from part (ii) of the same lemma. Thus, $v^{\Gamma}(S \cup T) \geq v^{\Gamma}(S)+v^{\Gamma}(T)$.

As the coalition functions of CTLD games yield the savings comparing the individual and aggregated costs, they are zero-normalized, that is for every $\Gamma \in \mathcal{T}$ and every $i \in N$ it holds that $v^{\Gamma}(\{i\})=0$. We discuss other properties of CTLD games in Sect. 7.

\section{CTLD solutions and their properties}

A CTLD allocation for a player set $N$ is a $|N|$-dimensional vector $\alpha=\left(\alpha_{i}\right)_{i \in N}$ with $\alpha_{i}$ being the allocation to player $i$. A CTLD solution is a set-valued function $A$ which for every CTLD situation $\Gamma \in \mathcal{T}$ with player set $N$ determines a set of allocations $A(\Gamma) \subseteq \mathbb{R}^{N}$. 
This definition of solution is innocuously different than the standard definition of solutions in cooperative game theory as our definition draws upon the situation rather than the game. Note that different situations with identical player sets can correspond to the same cooperative game. Thus, our definition of solution allows one to utilize information other than the savings obtained by different coalitions to devise allocations. The rest of this section is devoted to introducing desirable properties of CTLD solutions.

We start with properties which can also be expressed in relation to the cooperative games associated with CTLD situations. Perhaps the most intuitive desirable property of solutions in any cooperative situation is the efficiency property which requires that all the savings obtained in the grand coalition be completely divided among the players.

Property 1 A CTLD solution A satisfies the efficiency property if for every $\Gamma \in \mathcal{T}$ with player set $N$ and every $\alpha \in A(\Gamma)$ it holds that $\sum_{i \in N} \alpha_{i}=v^{\Gamma}(N)$.

The nonemptiness property defined below reflects the ability of a solution to produce at least one allocation in every given CTLD situation.

Property 2 A CTLD solution A satisfies the nonemptiness property (NE) iffor every $\Gamma \in \mathcal{T}, A(\Gamma)$ is non-empty.

A solution is inconclusive if it yields more than one allocation, if it could suggest any allocation at all. The uniqueness property addresses this issue.

Property 3 A CTLD solution A satisfies the uniqueness property $(U Q)$ if for every $\Gamma \in \mathcal{T}$ such that $A(\Gamma) \neq \emptyset$, it holds that $|A(\Gamma)|=1$.

The notion of stability is a critical concept in many cooperative situations, including CTLD situations. Given a CTLD situation $\Gamma \in \mathcal{T}$ with player set $N$ and $\in \in \mathbb{R}$, an allocation $\alpha$ is called $\epsilon$-stable if (a) $\alpha$ is efficient, i.e., $\sum_{i \in N} \alpha_{i}=v^{\Gamma}(N)$, and (b) for all $S \subset N$, it holds that $\sum_{i \in S} \alpha_{i}+\epsilon \geq v^{\Gamma}(S)$. The set of all $\epsilon$-stable allocations of the game associated with a situation comprises the $\epsilon$-core (Shapley and Shubik 1966). An $\epsilon$-stable allocation provides sufficient incentives for all players not to break apart from the grand coalition if reorganizing cooperation in a sub-coalition entails a cost of $\epsilon$ (or a bonus of $-\epsilon$ if $\epsilon<0$ ). The case of negative $\epsilon$ is of little practical importance in this paper since formation of cooperative organizations usually requires investment by the logistics providers. Thus, ideally a 0 -stable (or simply stable) allocation provides sufficient incentives for all players to remain in the grand coalition even if separating from the grand coalition is free. We will give an example in Sect. 7 to show that it may be impossible to find stable allocations in CTLD situations. The following property reflects the need for solutions that either produce stable allocations, or, when the latter cannot be achieved, obtain allocations that are as stable as possible.

Property 4 A CTLD solution A satisfies the least-instability property $(L U)$ iffor every $\Gamma \in \mathcal{T}$ with player set $N$ and every $\alpha \in A(\Gamma), \alpha$ is $\epsilon^{*}$-stable where

$$
\epsilon^{*}=\min \left\{\epsilon \geq 0 \mid \sum_{i \in S} \alpha_{i}+\epsilon \geq v^{\Gamma}(S) \text { for all } S \subset N, \sum_{i \in N} \alpha_{i}=v^{\Gamma}(N)\right\} \text {. }
$$



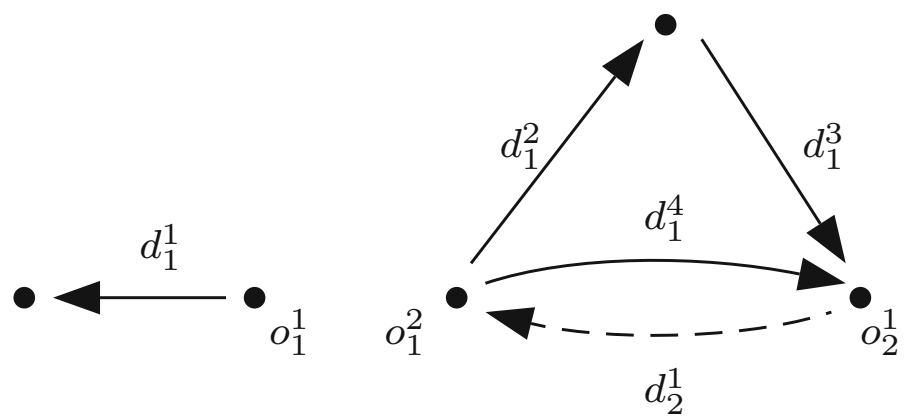

Fig. 3 Separable and irrelevant deliveries

The two properties introduced in the remainder of this section are specific to CTLD situations and address issues concerning the competitive positions of the players and the scope beyond which the network of deliveries of a player should be ignored by the solution. We start by introducing two special classes of delivery requirements in CTLD situations.

Definition 1 Let $\Gamma \in \mathcal{T}$ be a CTLD situation with player set $N . D \subseteq D_{i}$ is a separable delivery set (SDS) of player $i$ if

$$
c^{*}\left(O_{i}, D\right)+c^{*}\left(O_{N}, D_{N} \backslash D\right)=c^{*}\left(O_{N}, D_{N}\right) .
$$

Let $\operatorname{SDS}_{i}(\Gamma)$ be the set of SDSs of $i$.

The stand-alone cost of fulfilling a SDS of a player is additive to the cost of fulfilling the remaining deliveries in the grand coalition. Therefore, a player can individually fulfill a SDS of itself without disrupting the optimality of delivery plans in the grand coalition.

Example 3 Figure 3 depicts a CTLD situation $\Gamma$ with two players $N=\{1,2\}$. It is easy to see that player 1 can individually fulfill the delivery requirement $\left\{d_{1}^{1}\right\}$. Also, player 1 can take out either $\left\{d_{1}^{2}, d_{1}^{3}\right\}$ or $\left\{d_{1}^{4}\right\}$ (but not both!) from the grand coalition's delivery requirements and fulfill them separately such that the total cost of fulfillment does not increase. Thus, we have

$$
\operatorname{SDS}_{1}(\Gamma)=\left\{\left\{d_{1}^{1}\right\},\left\{d_{1}^{2}, d_{1}^{3}\right\},\left\{d_{1}^{4}\right\},\left\{d_{1}^{1}, d_{1}^{2}, d_{1}^{3}\right\},\left\{d_{1}^{1}, d_{1}^{4}\right\}\right\}
$$

The previous example shows two different types of SDSs in CTLD situations. While some SDSs of a player can be substituted with each other, there might exist SDSs which are separable in all scenarios. Therefore, among the SDSs of a player, we distinguish delivery sets which do not have any possible internal or external relevance to the rest of deliveries in any coalition.

Definition 2 Let $\Gamma \in \mathcal{T}$ be a CTLD situation with player set $N . D \subseteq D_{i}$ is an irrelevant delivery set (IDS) of $i$ if for all $D^{\prime} \subseteq D$, all $S \subseteq N$ with $i \in S$, and all $D^{\prime \prime} \subseteq D_{S} \backslash D$ it holds that 


$$
c^{*}\left(O_{i}, D^{\prime}\right)+c^{*}\left(O_{S}, D^{\prime \prime}\right)=c^{*}\left(O_{S}, D^{\prime} \cup D^{\prime \prime}\right) .
$$

Let $\operatorname{IDS}_{i}(\Gamma)$ be the set of IDSs of $i$.

The cost of fulfilling any subsets of irrelevant deliveries of a player is additive to any subset of the set of remaining deliveries in any coalition that includes that player, so the player can fulfill such deliveries separately in any possible combination with other deliveries. In Example 2, $\left\{d_{1}^{1}\right\}$ is the only IDS of player 1 . Note that neither $\left\{d_{1}^{2}, d_{1}^{3}\right\}$ nor $\left\{d_{1}^{4}\right\}$ remains separable if player 1 removes the other set and plan its delivery by himself. This example clarifies that every IDS is also separable, but the reverse does not hold necessarily.

We are ready to present the first property in this section which specifies a scope of consideration for CTLD situations where the delivery requirements beyond this scope should be ignored in the calculation of allocations. We define the IID property as the insensitivity of a solution to the exclusion of irrelevant deliveries of the players. Given $D_{i}^{\prime} \subseteq D_{i}$, let $\Gamma \backslash D_{i}^{\prime}$ be a CTLD situation that coincides with $\Gamma$ except for the delivery set of $i$ which is replaced by $D_{i} \backslash D_{i}^{\prime}$.

Property 5 A CTLD solution A satisfies the IID property if for every $\Gamma \in \mathcal{T}$ with player set $N$, any $i \in N$, and every $D \in \operatorname{IDS}_{i}(\Gamma)$ it holds that $A(\Gamma)=A(\Gamma \backslash D)$.

The final property in this section addresses the competitive aspect of solutions in CTLD situations. Considering the limited number of players in consortia of logistics providers and the competitive nature of transportation markets, a key requirement for solutions in CTLD situations is their ability to maintain the competitive positions of the players in dividing the savings obtained by cooperation.

Recall from Sect. 3 that the average cost of fulfillment represents the lowest price that $i$ can charge for every unit distance of its delivery services. In this regard, the average cost of fulfillment provides a basis for calculating unit delivery prices in logistics markets. However, it can also be utilized as a measure of comparison among the players. This idea is motivated by the observation that a lower average cost of fulfillment of a logistics player compared to that of another logistics player allows the former to charge a lower unit price for its delivery services while remaining profitable. Therefore, if for two players $i$ and $j$ it holds that $z\left(O_{i}, D_{i}\right)<z\left(O_{j}, D_{j}\right)$, it can be stated that prior to cooperation, $i$ is in a better competitive position than $j$. The definition of average cost of fulfillment can be naturally extended to incorporate the savings allocated to the players after the cooperation. Given an allocation $\alpha$ and player $i \in N, D_{i} \neq \emptyset$, define the average cost of fulfillment of a player $i$ under $\alpha$ as

$$
z_{i}^{\alpha}\left(O_{i}, D_{i}\right)=\frac{c^{*}\left(O_{i}, D_{i}\right)-\alpha_{i}}{c_{F}\left(D_{i}\right)} .
$$

Note that the cost of full kilometers of any non-empty set of delivery requirements is strictly positive. The notion of competitiveness in this paper is motivated by the observation that non-competitive allocations eliminate the advantage of a player over other players in terms of its competitive position before and after cooperation. We elaborate with the help of an example. 


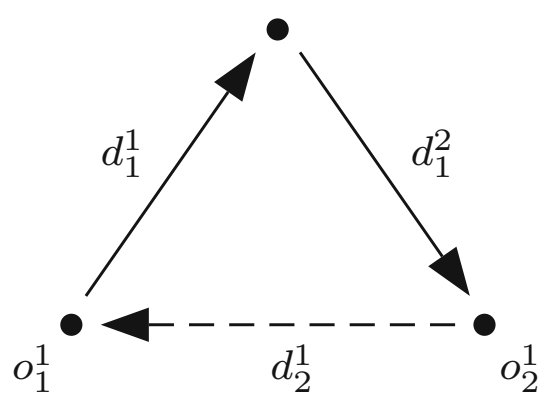

Fig. 4 A CTLD situation where players have different competitive positions

Example 4 Figure 4 represents a CTLD situation with two players $N=\{1,2\}$. Assuming that the distance between any two locations is 1 , we get $z\left(O_{1}, D_{1}\right)=1.5$ and $z\left(O_{2}, D_{2}\right)=2$. The cooperation in this case results in two units of savings, i.e., $v^{\Gamma}(N)=2$. Observe that the equal allocation $\alpha=(1,1)$ results in having $z_{1}^{\alpha}\left(O_{1}, D_{1}\right)=z_{2}^{\alpha}\left(O_{2}, D_{2}\right)=1$. Thus, the equal allocation eliminates player 1's advantage over player 2 with regard to their competitive positions prior to the cooperation.

We are now ready to introduce a competitiveness property defined over a restricted set of CTLD situations. Let $\hat{\mathcal{T}}$ be the set of every CTLD situation $\Gamma \in \mathcal{T}$ with player set $N$ such that $\operatorname{SDS}_{i}(\Gamma)=\{\emptyset\}$ for all $i \in N$.

Property 6 A CTLD solution satisfies the restricted competitiveness property $(R C)$ if for every situation $\Gamma \in \hat{\mathcal{T}}$ with player set $N=\{1,2\}$ and any $\alpha \in A(\Gamma)$ it holds that

$$
z_{1}^{\alpha}\left(O_{1}, D_{1}\right) z\left(O_{2}, D_{2}\right)=z_{2}^{\alpha}\left(O_{2}, D_{2}\right) z\left(O_{1}, D_{1}\right)
$$

If the set of delivery requirements of every player is non-empty, then (11) boils down to $z\left(O_{1}, D_{1}\right) / z_{1}^{\alpha}\left(O_{1}, D_{1}\right)=z\left(O_{2}, D_{2}\right) / z_{2}^{\alpha}\left(O_{2}, D_{2}\right)$. In this respect, the RC property prescribes allocations that preserve the competitive positions of the players, that is, an allocation satisfying the RC property equalizes the ratio of average costs of fulfillments of the players before and after cooperation in two-player reductions of the original situations. Note that the latter can also be expressed in terms of the stand-alone costs of the players, i.e., for players with non-empty delivery sets (11) simplifies to $\alpha_{1} / c^{*}\left(O_{1}, D_{1}\right)=\alpha_{2} / c^{*}\left(O_{2}, D_{2}\right)$.

The RC property is defined in terms of situations wherein the SDSs of all players are empty, i.e., no player has any separable delivery requirements. This allows us to postpone the treatment of SDSs until the introduction of our proposed solution. One can extend the definition of property 6 to situations with any number of players. However, to avoid impossibility issues regarding the preservation of all properties simultaneously, we stated the competitiveness property in a restricted manner and in terms of two-player situations. In Example 4, the allocation $\alpha=(1.2,0.8)$ preserves the competitive positions of players 1 and 2 before and after the cooperation, resulting in $z_{1}^{\alpha}\left(O_{1}, D_{1}\right)=0.9$ and $z_{2}^{\alpha}\left(O_{2}, D_{2}\right)=1.2$. 


\section{A solution for CTLD situations}

In this section, we introduce a CTLD solution that satisfies all the properties mentioned above. Our solution draws upon essential delivery sets, i.e., particular subsets of deliveries of a player which are necessary and sufficient in creating its contribution to the grand coalition.

\subsection{Essential delivery sets}

Definition 3 Let $\Gamma \in \mathcal{T}$ be a CTLD situation with player set $N . D \subseteq D_{i}$ is an essential delivery set (EDS) of player $i$ if

$$
c^{*}\left(O_{i}, D_{i} \backslash D\right)+c^{*}\left(O_{N}, D_{N \backslash i} \cup D\right)=c^{*}\left(O_{N}, D_{N}\right) .
$$

and for every $D^{\prime} \subset D, D \neq \emptyset$ :

$$
c^{*}\left(O_{i}, D_{i} \backslash D^{\prime}\right)+c^{*}\left(O_{N}, D_{N \backslash i} \cup D^{\prime}\right)>c^{*}\left(O_{N}, D_{N}\right) .
$$

Let $\operatorname{EDS}_{i}(\Gamma)$ be the set of all essential delivery sets of $i$.

An essential delivery set of a player $i$ meets two conditions. First, the complement of this set comprises a SDS of player $i$, that is, an essential delivery set is sufficient for creating the cost savings of players in the grand coalition. Second, one cannot expand the complement of this set to obtain a larger SDS for player $i$. In fact, an essential delivery set is necessary for creating a player's contribution to the grand coalition in the sense that the situation obtained by excluding its complement delivery set contains no SDSs. The following lemma formalizes this.

Lemma 3 Let $\Gamma \in \mathcal{T}$ be a CTLD situation with player set $N$. Let $i \in N, D \in$ $\operatorname{EDS}_{i}(\Gamma)$, and $\Gamma^{\prime}=\Gamma \backslash\left(D_{i} \backslash D\right)$. We have $\operatorname{SDS}_{i}\left(\Gamma^{\prime}\right)=\emptyset$.

Proof It suffices to show that for any $D^{*} \subseteq D$ it holds that

$$
c^{*}\left(O_{i}, D^{*}\right)+c^{*}\left(O_{N}, D_{N \backslash i} \cup\left(D \backslash D^{*}\right)\right)>c^{*}\left(O_{N}, D_{N \backslash i} \cup D\right) .
$$

For any $D^{*} \subseteq D$ we have

$$
\begin{aligned}
c^{*} & \left(O_{i}, D^{*}\right)+c^{*}\left(O_{N}, D_{N \backslash i} \cup\left(D \backslash D^{*}\right)\right) \\
& =c^{*}\left(O_{i}, D^{*}\right)+c^{*}\left(O_{N}, D_{N \backslash i} \cup\left(D \backslash D^{*}\right)\right)+c^{*}\left(O_{i}, D_{i} \backslash D\right)-c^{*}\left(O_{i}, D_{i} \backslash D\right) \\
& \geq c^{*}\left(O_{i}, D_{i} \backslash\left(D \backslash D^{*}\right)\right)+c^{*}\left(O_{N}, D_{N \backslash i} \cup\left(D \backslash D^{*}\right)\right)-c^{*}\left(O_{i}, D_{i} \backslash D\right) \\
& >c^{*}\left(O_{N}, D_{N}\right)-c^{*}\left(O_{i}, D_{i} \backslash D\right) \\
& =c^{*}\left(O_{N}, D_{N \backslash i} \cup D\right) .
\end{aligned}
$$

where the first inequality follows from part (i) of Lemma 1, the second inequality follows from the second condition of EDS in (13) for $D^{\prime}=D \backslash D^{*}$, and the last 
equality follows from the first condition of EDS in (12). Therefore, Eq. (14) holds for any $D^{*} \subseteq D$.

In Example 3 (Fig. 3), player 1 has two sets of essential delivery sets $\left\{d_{1}^{2}, d_{1}^{3}\right\}$ and $\left\{d_{1}^{4}\right\}$. This demonstrates that a player in a CTLD situation might have multiple essential delivery sets.

The next lemma elaborates on the relation between the essential and the IDSs.

Lemma 4 Let $\Gamma \in \mathcal{T}$ be a CTLD situation with player set $N$. Let $i \in N$ and $D_{i}^{r} \in$ $\operatorname{IDS}_{i}(\Gamma)$. Then

(i) for every $D \in \operatorname{EDS}_{i}(\Gamma), D \cap D_{i}^{r}=\emptyset$,

(ii) for every $j \in N, \operatorname{EDS}_{j}(\Gamma)=\operatorname{EDS}_{j}\left(\Gamma \backslash D_{i}^{r}\right)$.

Proof (i) Suppose the contrary, that is $D \cap D_{i}^{r} \neq \varnothing$. Let $D^{\prime}=D \cap D_{i}^{r}$, hence $D^{\prime} \subseteq D$. By definition of irrelevant deliveries of player $i$ in $\Gamma$ it must be that

$$
c^{*}\left(O_{i}, D^{\prime}\right)+c^{*}\left(O_{N}, D_{N \backslash i} \cup\left(D \backslash D^{\prime}\right)\right)=c^{*}\left(O_{N}, D_{N \backslash i} \cup D\right) \text {. }
$$

The latter implies that $D^{\prime}$ is a SDS of player $i$ in $\Gamma \backslash\left(D_{i} \backslash D\right)$ which contradicts Lemma 3. Thus, it must be that $D \cap D_{i}^{r}=\emptyset$.

(ii) First we show that for any $j \in N$ and every $D^{\prime} \subseteq D_{N} \backslash D_{i}^{r}$ such that $D^{\prime} \neq \emptyset$ it holds that

$$
\begin{aligned}
& c^{*}\left(O_{j}, D_{j} \backslash D^{\prime}\right)+c^{*}\left(O_{N}, D_{N \backslash j} \cup D^{\prime}\right)-c^{*}\left(O_{N}, D_{N}\right) \\
& \quad=c^{*}\left(O_{j}, D_{j} \backslash D^{\prime}\right)+c^{*}\left(O_{N},\left(D_{N \backslash j} \backslash D_{i}^{r}\right) \cup D^{\prime}\right)-c^{*}\left(O_{N}, D_{N} \backslash D_{i}^{r}\right) .
\end{aligned}
$$

Since $D_{i}^{r} \in \operatorname{IDS}_{i}(\Gamma)$, we have

$$
c^{*}\left(O_{i}, D_{i}^{r}\right)+c^{*}\left(O_{N}, D_{N} \backslash D_{i}^{r}\right)=c^{*}\left(O_{N}, D_{N}\right),
$$

and, since $\left(D_{N \backslash j} \backslash D_{i}^{r}\right) \cup D^{\prime} \subset D_{N} \backslash D_{i}^{r}$, we have

$$
c^{*}\left(O_{i}, D_{i}^{r}\right)+c^{*}\left(O_{N},\left(D_{N \backslash j} \backslash D_{i}^{r}\right) \cup D^{\prime}\right)=c^{*}\left(O_{N}, D_{N \backslash j} \cup D^{\prime}\right) .
$$

By (17) and (18) we get

$$
\begin{aligned}
c^{*}\left(O_{N}, D_{N \backslash j} \cup D^{\prime}\right)-c^{*}\left(O_{N}, D_{N}\right)= & c^{*}\left(O_{N},\left(D_{N \backslash j} \backslash D_{i}^{r}\right) \cup D^{\prime}\right) \\
& -c^{*}\left(O_{N}, D_{N} \backslash D_{i}^{r}\right) .
\end{aligned}
$$

Adding $c^{*}\left(O_{j}, D_{j} \backslash D^{\prime}\right)$ to both sides of the Eq. (19) obtains Eq. (16).

Suppose that $D \in \operatorname{EDS}_{j}(\Gamma)$. For $j \neq i$ it holds that $D \subseteq D_{j}$, and for $j=i$ part (i) of this lemma indicates that $D \subseteq D_{i} \backslash D_{i}^{r}$. Therefore, for $j \in N$ we have $D \subseteq D_{N} \backslash D_{i}^{r}$. By definition of EDS it must be that

$$
c^{*}\left(O_{j}, D_{j} \backslash D\right)+c^{*}\left(O_{N}, D_{N \backslash j} \cup D\right)=c^{*}\left(O_{N}, D_{N}\right)
$$


and for every $D^{\prime \prime} \subset D, D^{\prime \prime} \neq \varnothing$,

$$
c^{*}\left(O_{j}, D_{j} \backslash D^{\prime \prime}\right)+c^{*}\left(O_{N}, D_{N \backslash j} \cup D^{\prime \prime}\right)>c^{*}\left(O_{N}, D_{N}\right) .
$$

However, due to (16), equality in (20) implies that

$$
c^{*}\left(O_{j}, D_{j} \backslash D\right)+c^{*}\left(O_{N},\left(D_{N \backslash j} \backslash D_{i}^{r}\right) \cup D\right)=c^{*}\left(O_{N}, D_{N} \backslash D_{i}^{r}\right)
$$

and, due to (16), inequality in (21) implies that

$$
c^{*}\left(O_{j}, D_{j} \backslash D^{\prime \prime}\right)+c^{*}\left(O_{N},\left(D_{N \backslash j} \backslash D_{i}^{r}\right) \cup D^{\prime \prime}\right)>c^{*}\left(O_{N}, D_{N} \backslash D_{i}^{r}\right)
$$

for every $D^{\prime \prime} \subset D, D^{\prime \prime} \neq \emptyset$. The conditions in (22) and (23) indicate that $D \in \operatorname{EDS}_{j}\left(\Gamma \backslash D_{i}^{r}\right)$.

Suppose that $D \in \operatorname{EDS}_{j}\left(\Gamma \backslash D_{i}^{r}\right)$. For $j \neq i$ it holds that $D \subseteq D_{j}$, and for $j=i$ it is the case that $D \subseteq D_{i} \backslash D_{i}^{r}$. Therefore, for $j \in N$ we have $D \subseteq D_{N} \backslash D_{i}^{r}$. By definition of EDS equality (22) as well as inequality (23) for every $D^{\prime \prime} \subset D$, $D^{\prime \prime} \neq \emptyset$, hold. As direct results of (16), equality (20) as well as inequality (21) for every $D^{\prime \prime} \subset D, D^{\prime \prime} \neq \emptyset$, must also hold. We conclude that $D \in \operatorname{EDS}_{j}(\Gamma)$.

Part (i) of Lemma 4 asserts that the essential delivery sets never include any irrelevant deliveries. Part (ii) shows that the exclusion of irrelevant deliveries of any player from the entire delivery set of the grand coalition does not affect the sets of essential delivery sets of any player.

The last lemma in this section shows that for finding essential delivery sets, it is sufficient to compare the optimal individual plans and those of the grand coalition. Given an optimal plan of a player $i$ and an optimal plan for the grand coalition, an essential delivery set of $i$ comprises the delivery requirements whose fulfillment in the grand coalition involve other players, i.e., they are either fulfilled from depots of other players or in trips which contain delivery requirements of players other than $i$.

Lemma 5 Let $\Gamma \in \mathcal{T}$ be a CTLD situation with player set $N$. Let $i \in N$ and $D \in$ $\operatorname{EDS}_{i}(\Gamma)$. There exists $P \in \mathcal{P}^{*}\left(O_{N}, D_{N}\right)$ such that $D=\bigcup_{l \in P \backslash L\left(O_{i}, D_{i}\right)} D^{l}$.

Proof Let $P^{\prime} \in \mathcal{P}^{*}\left(O_{i}, D_{i} \backslash D\right), P^{\prime \prime} \in \mathcal{P}^{*}\left(O_{N}, D_{N \backslash i} \cup D\right)$, and $P=P^{\prime} \cup P^{\prime \prime}$. By first condition of EDS in (12) we have $c^{*}\left(O_{i}, D_{i} \backslash D\right)+c^{*}\left(O_{N}, D_{N \backslash i} \cup D\right)=c^{*}\left(O_{N}, D_{N}\right)$. By Lemma 2, we have $P \in \mathcal{P}^{*}\left(O_{N}, D_{N}\right)$. Note that $P^{\prime} \subseteq L\left(O_{i}, D_{i}\right)$. To complete the proof it suffices to show that $P^{\prime \prime} \cap L\left(O_{i}, D_{i}\right)=\emptyset$. Suppose the contrary. Then there must exist trip $l$ such that $l \in P^{\prime \prime} \cap L\left(O_{i}, D_{i}\right)$ which requires that $D^{l} \subset D \subseteq D_{i}$. As the cost of delivery plan $P^{\prime \prime}$ is the sum of its individual trips, it must be that

$$
c^{l}+c^{*}\left(O_{N}, D_{N \backslash i} \cup\left(D \backslash D^{l}\right)\right)=c^{*}\left(O_{N}, D_{N \backslash i} \cup D\right) .
$$

Since $l \in L\left(O_{i}, D_{i}\right)$, we have $c^{l}=c^{*}\left(O_{i}, D^{l}\right)$. Then it must be that

$$
c^{*}\left(O_{i}, D^{l}\right)+c^{*}\left(O_{N}, D_{N \backslash i} \cup\left(D \backslash D^{l}\right)\right)=c^{*}\left(O_{N}, D_{N \backslash i} \cup D\right) .
$$


The latter implies that $D^{l}$ is a SDS of $i$ in $\left(\Gamma \backslash D_{i}\right) \cup D$ which contradicts Lemma 3 . Thus, given $D$, any optimal fulfillment plan of the form $P=P^{\prime} \cup P^{\prime \prime}$ has the feature that $D=\bigcup_{l \in P \backslash L\left(O_{i}, D_{i}\right)} D^{l}$.

In light of the previous lemma, the following procedure obtains the essential delivery sets of a player $i$. Given the CTLD situation, suppose that the set of optimal plans are available. Choose an optimal plan and find every trip whose deliveries as well as its depot entirely belong to $i$. An essential delivery set of $i$ is obtained by excluding the deliveries in latter trips from $i$ 's delivery set for this plan. By repeating this procedure for all optimal plans the set of essential delivery sets of $i$ is identified.

Given the favorable features of essential delivery sets, we concentrate on them when determining players allocations in CTLD situations. However, as seen in Example 3 , the essential delivery sets of a player can be multiple. In those cases, we focus on essential delivery sets which have the lowest costs when fulfilled individually. In this way, we make sure that players fulfill the most costly parts of their delivery requirements by themselves. We introduce the minimal essential delivery sets (MEDS) as the essential delivery sets with minimum stand-alone cost.

Definition 4 Let $\Gamma \in \mathcal{T}$ be a CTLD situation with player set $N . D \subseteq D_{i}$ is a MEDS of $i$ if $D \in \operatorname{EDS}_{i}(\Gamma)$ and

$$
c^{*}\left(O_{i}, D\right) \leq c^{*}\left(O_{i}, D^{\prime}\right) \quad \text { for all } D^{\prime} \in \operatorname{EDS}_{i}(\Gamma)
$$

Let $\operatorname{MEDS}_{i}(\Gamma)$ be the set of MEDSs of $i$.

Note that even if the set of MEDSs has multiple elements, the stand-alone costs of fulfillment for its elements are equal.

The set of MEDSs of a player $i$ can be obtained by comparing among its essential delivery sets to find the essential delivery sets that have the minimum costs of delivery when fulfilled separately from the depots of $i$.

\subsection{The proposed solution}

Our proposed CTLD solution is introduced in two steps. In the first step, we introduce a proportional CTLD solution, $A^{P}$, which incorporates the notions of competitiveness and scope defined in the previous section. In the second step, we use the latter proportional allocation to construct a least-unstable solution, $A^{E}$.

Fix $\Gamma$, let $D_{i}^{m} \in \operatorname{MEDS}_{i}(\Gamma)$, and define $A^{P}(\Gamma)=\left\{\alpha^{P}(\Gamma)\right\}$ such that

$$
\alpha_{i}^{P}(\Gamma)= \begin{cases}\frac{c^{*}\left(O_{i}, D_{i}^{m}\right)}{\sum_{j \in N} c^{*}\left(O_{j}, D_{j}^{m}\right)} v^{\Gamma}(N) & \text { if } \sum_{j \in N} c^{*}\left(O_{j}, D_{j}^{m}\right) \neq 0 \\ \frac{1}{n} v^{\Gamma}(N) & \text { otherwise. }\end{cases}
$$

When there exists at least one player with a non-empty essential delivery set, the solution $A^{P}$ obtains a unique efficient allocation that divides the savings obtained in the grand coalition of CTLD situation $\Gamma$ among players with non-empty essential 


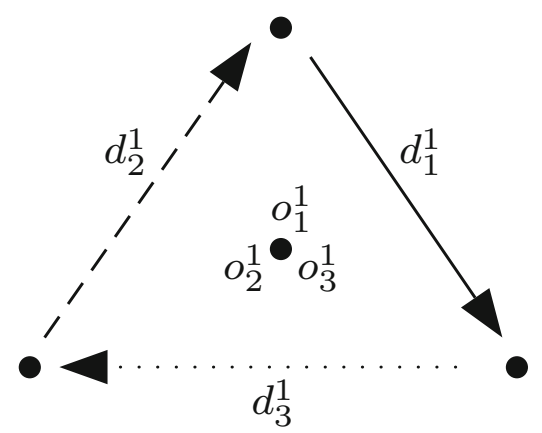

Fig. 5 A CTLD situation with empty EDS for all players

delivery sets proportional to the stand-alone cost of their minimal essential deliveries. The CTLD solution $A^{P}$ completely preserves the competitive positions of the players with regard to their MEDSs. This means that for every pair of players $i, j \in N$ with non-empty essential delivery sets we have $\alpha_{i}^{P}(\Gamma) / c^{*}\left(O_{i}, D_{i}^{m}\right)=\alpha_{j}^{P}(\Gamma) / c^{*}\left(O_{j}, D_{j}^{m}\right)$ which implies that

$$
\frac{z_{i}\left(O_{i}, D_{i}^{m}\right)}{z_{i}^{\alpha^{P}(\Gamma)}\left(O_{i}, D_{i}^{m}\right)}=\frac{z_{j}\left(O_{j}, D_{j}^{m}\right)}{z_{j}^{\alpha^{P}(\Gamma)}\left(O_{j}, D_{j}^{m}\right)} .
$$

If the set of essential delivery sets of every player is empty, which results in having $\sum_{j \in N} c^{*}\left(O_{j}, D_{j}^{m}\right)=0$, then $A^{P}$ allocates the savings among the players equally. This situation could happen when the delivery requirements of players that generate the savings in the grand coalition are completely substitutable with deliveries of some other players. The following example presents such a situation.

Example 5 Consider the CTLD situation $\Gamma$ depicted in Fig. 5. There are three players $N=\{1,2,3\}$ each having a depot and a delivery requirement. The distance between the pickup and delivery locations for all delivery requirements is two and the distance from the depots to any pickup/delivery point is one. The set of feasible trips includes all trips which fulfill no more than two delivery requirements, i.e., $L=\left\{l \in \mathcal{L}|| D^{l} \mid \leq 2\right\}$ (only two deliveries can be fulfilled sequentially during a day). The savings obtained in the grand coalition are only as much as that in any two-player coalition thus the inclusion of the third player, arbitrarily chosen among the three, does not add to the total savings. Therefore, we have $\operatorname{EDS}_{1}(\Gamma)=\operatorname{EDS}_{2}(\Gamma)=\operatorname{EDS}_{3}(\Gamma)=\{\emptyset\}$.

The solution $A^{P}$ does not necessarily obtain an $\epsilon^{*}$-stable allocation. To achieve this, we present the stable CTLD solution $A^{E}$. Define $A^{E}(\Gamma)$ such that

$$
\begin{aligned}
& A^{E}(\Gamma)=\underset{\alpha \in \mathbb{R}^{N}}{\arg \min } \sum_{i \in N}\left(\alpha_{i}^{P}(\Gamma)-\alpha_{i}\right)^{2} \\
& \text { s.t. } \quad \sum_{i \in S} \alpha_{i}+\epsilon^{*} \geq v^{\Gamma}(S) \quad \forall S \subset N \\
& \sum_{i \in N} \alpha_{i}=v^{\Gamma}(N)
\end{aligned}
$$


where $\epsilon^{*}$ is defined in (7). Given the situation $\Gamma$, the solution $A^{E}(\Gamma)$ gives the set of all $\epsilon^{*}$-stable allocations that have the shortest distance from the proportional allocation $\alpha_{i}^{P}(\Gamma)$.

Theorem 2 The solution $A^{E}$ satisfies the nonemptiness, uniqueness, and leastinstability properties.

Proof Let $\Gamma \in \mathcal{T}$ be a CTLD situation with player set $N$. We proceed in order.

NE By the definition of feasible trip sets, $v^{\Gamma}$ is well defined. From (25) it is clear that $\alpha^{P}(\Gamma)$ always exists since either $\sum_{j \in N} c^{*}\left(O_{j}, D_{j}^{m}\right) \neq 0$ or $\sum_{j \in N} c^{*}\left(O_{j}, D_{j}^{m}\right)=0$. Also, definition of $\epsilon^{*}$ guarantees the existence of $\alpha$ that satisfies (27) and (28) (Maschler et al. 1979). Therefore, $A^{E}(\Gamma) \neq \emptyset$. We conclude that $A^{E}$ satisfies the nonemptiness property.

UQ The allocations contained in $A^{E}(\Gamma)$ minimize the Euclidean distance between $\alpha^{P}(\Gamma)$ and the set of $\epsilon^{*}$-stable allocations defined via (27) and (28). Note that the region defined via (27) and (28), which is essentially an $\epsilon$-core, is a compact convex polytope (Maschler et al. 1979). The convex projection theorem (Davidson and Donsig 2010) asserts that there exists a unique point in every non-empty closed and convex set having the minimum Euclidean distance from any given point. Therefore, $\left|A^{E}(\Gamma)\right|=1$ which implies that $A^{E}$ satisfies the uniqueness property.

LU The unique allocation obtained by $A^{E}(\Gamma)$ satisfies the constraints in (27) and (28). By definition of $\epsilon^{*}$-stability in (7), this allocation is an $\epsilon^{*}$-stable allocation as well. It follows that the solution $A^{E}$ satisfies the LU property.

Since the allocation $A^{E}$ satisfies the UQ property, in any CTLD situation $\Gamma$ it results in a single allocation. We denote this single allocation with $\alpha^{E}(\Gamma)$, i.e., $A^{E}(\Gamma)=$ $\left\{\alpha^{E}(\Gamma)\right\}$.

Before providing the results regarding the ability of $A^{E}$ to satisfy IID and RC properties, we show that the coalition functions in CTLD situations remain intact if IDSs of the players are excluded from the situation.

Lemma 6 Let $\Gamma \in \mathcal{T}$ be a CTLD situation with player set $N$. Let $i \in N$ and $D \in$ $\operatorname{IDS}_{i}(\Gamma)$. We have $v^{\Gamma}(S)=v^{\Gamma \backslash D}(S)$ for every $S \subseteq N$.

Proof From the definition of IDSs we know that

$$
c^{*}\left(O_{i}, D\right)+c^{*}\left(O_{i}, D_{i} \backslash D\right)=c^{*}\left(O_{i}, D_{i}\right)
$$

and for $S \subseteq N, i \in S$,

$$
c^{*}\left(O_{i}, D\right)+c^{*}\left(O_{S}, D_{S} \backslash D\right)=c^{*}\left(O_{S}, D_{S}\right)
$$

By definition of $v^{\Gamma}$ it then follows that

$$
v^{\Gamma}(S)=\sum_{j \in S \backslash i} c^{*}\left(O_{j}, D_{j}\right)+c^{*}\left(O_{i}, D_{i}\right)-c^{*}\left(O_{S}, D_{S}\right)
$$




$$
\begin{aligned}
& =\sum_{j \in S \backslash i} c^{*}\left(O_{j}, D_{j}\right)+c^{*}\left(O_{i}, D_{i} \backslash D\right)-c^{*}\left(O_{S}, D_{S} \backslash D\right) \\
& =v^{\Gamma \backslash D}(S) .
\end{aligned}
$$

In light of the previous lemma, it can be inferred that the CTLD game associated with a CTLD situation remains the same after excluding the IDSs of players.

Next theorem asserts that $A^{E}$ also satisfies the remaining two properties defined specifically for CTLD situations.

Theorem 3 The solution $A^{E}$ satisfies the IID and restricted competitiveness properties.

Proof Let $\Gamma \in \mathcal{T}$ be a CTLD situation with player set $N$.

IID Let $D_{i}^{r} \in \operatorname{IDS}_{i}(\Gamma)$. By Lemma 6 , we have $v^{\Gamma}(S)=v^{\Gamma \backslash D_{i}^{r}}(S)$ for all $S \subseteq N$. Thus, the constraints in (27) and (28) would not be affected by exclusion of the irrelevant deliveries. It remains to show that $\alpha_{j}^{P}(\Gamma)=\alpha_{j}^{P}\left(\Gamma \backslash D_{i}^{r}\right)$ for every $j \in N$. By part (ii) of Lemma 4 we know that $\operatorname{EDS}_{j}(\Gamma)=\operatorname{EDS}_{j}\left(\Gamma \backslash D_{i}^{r}\right)$ for all $j \in N$. Consequently, $\operatorname{MEDS}_{j}(\Gamma)=\operatorname{MEDS}_{j}\left(\Gamma \backslash D_{i}^{r}\right)$ for all $j \in N$. From the definition of $\alpha^{P}$ in (25) in conjunction with Lemma 6 it immediately follows that $\alpha_{j}^{P}(\Gamma)=\alpha_{j}^{P}\left(\Gamma \backslash D_{i}^{r}\right)$ for every $j \in N$. Thus, $A^{E}(\Gamma)=A^{E}\left(\Gamma \backslash D_{i}^{r}\right)$ which proves that $A^{E}$ satisfies the IID property.

RC Suppose $N=\{1,2\}$. In this case the constraints in (27) and (28) are reduced to $\alpha_{1}+\alpha_{2}=v^{\Gamma}(N)$ and $\alpha_{1}, \alpha_{2} \geq 0$. The allocation $\alpha^{P}(\Gamma)$ is within this region. Hence, $\alpha^{E}(\Gamma)=\alpha^{P}(\Gamma)$ which results in the objective function value of zero in (26). Next suppose that $\operatorname{SDS}_{1}(\Gamma)=\operatorname{SDS}_{2}(\Gamma)=\emptyset$. In this case, we have $\operatorname{MEDS}_{i}(\Gamma)=\operatorname{EDS}_{i}(\Gamma)=\left\{D_{i}\right\}$ for $i \in N$. By definition of $\alpha^{P}(\Gamma)$ we get $\alpha_{1}^{P}(\Gamma) / c^{*}\left(O_{1}, D_{1}\right)=\alpha_{2}^{P}(\Gamma) / c^{*}\left(O_{2}, D_{2}\right)$ which implies that (11) holds. Therefore, the solution $A^{E}$ satisfies the RC property.

The solution $A^{E}$ incorporates the notions of scope and competitiveness to produce allocations for CTLD situations. When the proportional solution $A^{P}$ is within the set of $\epsilon^{*}$-stable allocations, defined via (27) and (28), $A^{E}$ coincides with $A^{P}$. Otherwise, when the allocation obtained by $A^{P}$ is not an $\epsilon^{*}$-stable allocation, $A^{E}$ draws upon a simple mechanism to single out an allocation in relation to the $A^{P}$.

Obtaining the solution $A^{E}$ requires solving a quadratic optimization problem to find the minimum Euclidean norm of a convex polytope. In this context, the early work of Wolfe (1976) provides several basic results, gives an algorithm based on the simplicial decomposition of the convex polytope, and proves its convergence. Despite its fast convergence, it is open to determine whether the Wolfe algorithm runs in polynomial time in dimension of space (Fujishige and Isotani 2011). Nevertheless, the limited number of players participating in practical instances of CTLD situations allows one to rely on generic solvers to calculate the proposed solution. 
Table 1 Comparing different solutions in CTLD situations

\begin{tabular}{lllllll}
\hline & Solution & RC & IID & NE & UQ & LU \\
\hline$A^{\mathcal{C}}$ & Core & $\times$ & $\checkmark$ & $\times$ & $\times$ & $\checkmark$ \\
$A^{\mathcal{L C}}$ & Least-core & $\times$ & $\checkmark$ & $\checkmark$ & $\times$ & $\checkmark$ \\
$A^{\eta}$ & Nucleolus & $\times$ & $\checkmark$ & $\checkmark$ & $\checkmark$ & $\checkmark$ \\
$A^{\Phi}$ & Shapley value & $\times$ & $\checkmark$ & $\checkmark$ & $\checkmark$ & $\times$ \\
$A^{A C A}$ & ACA & $\times$ & $\checkmark$ & $\times$ & $\checkmark$ & $\times$ \\
$A^{\psi}$ & Proportional & $\checkmark$ & $\times$ & $\times$ & $\checkmark$ & $\times$ \\
$A^{\text {EPM }}$ & EPM & $\checkmark$ & $\times$ & $\times$ & $\times$ & $\checkmark$ \\
$A^{E}$ & Proposed & $\checkmark$ & $\checkmark$ & $\checkmark$ & $\checkmark$ & $\checkmark$ \\
\hline$R C$ & & $\checkmark$ & & $\checkmark$ & \\
\hline
\end{tabular}

$R C$ restricted competitiveness, $I I D$ independence of irrelevant deliveries, $N E$ nonemptiness, $U Q$ uniqueness, $L U$ least-instability

\section{Adopting existing solutions for CTLD situations}

This section discusses the adoption of some of the existing solutions for CTLD situations and compares them with regard to the properties introduced earlier in this paper. Table 1 at the end of this section exhibits the summary of the results.

One of best-known solutions in cooperative game theory is the core (Gillies 1959). The core of a cooperative game contains all allocations that are efficient and stable. In this regard, the core in itself is an $\epsilon$-core with $\epsilon=0$. The core of a CTLD situation $\Gamma$ with player set $N$ can be defined accordingly as a mapping that assign to every CTLD situation the core of its associated game:

$$
A^{\mathcal{C}}(\Gamma)=\left\{\alpha \in \mathbb{R}^{N} \mid \sum_{i \in S} \alpha_{i} \geq v^{\Gamma}(S) \text { for all } S \subset N \text {, and } \sum_{i \in N} \alpha_{i}=v^{\Gamma}(N)\right\} .
$$

The core of CTLD situations does not satisfy the NE property. Example 5 in Sect. 6.2 depicts a CTLD situation where the latter is the case. In that example, for $S \subseteq N$ we have $c^{*}\left(O_{S}, D_{S}\right)=4$ if $|S|=1, c^{*}\left(O_{S}, D_{S}\right)=6$ if $|S|=2$, and $c^{*}\left(O_{N}, D_{N}\right)=10$. This results in $v^{\Gamma}(S)=0$ when $|S|=1, v^{\Gamma}(S)=2$ when $|S|=2$, and $v^{\Gamma}(N)=2$. No efficient and stable allocation can be found in this setting thus the core is empty. Upon existence, the core of a CTLD situation could as well contain an infinite number of allocations. For example, in every two-player CTLD situations with $v^{\Gamma}(N)>0$, any efficient allocation that gives non-negative allocations to the players is in the core. Therefore, the core of CTLD situations does not satisfy the UQ property. On the other hand, the definition of RC property in combination with the efficiency property necessitates a unique allocation in every two-player situation. Consequently, the core does not satisfy the RC property either. Since the core solely draws upon the CTLD game, it follows from Lemma 6 that it satisfies the IID property.

The intuitive appeal of the stability concept on one side, and the possibility of having empty cores on the other side motivates alternative solutions that address the stability issue. The least-core of a game (Maschler et al. 1979) is the intersection of 
all non-empty $\epsilon$-cores of it. Accordingly, the least-core of a CTLD situation is defined as a mapping that assigns to every situation $\Gamma \in \mathcal{T}$ with player set $N$ the least-core of its associated game:

$$
A^{\mathcal{L C}}(\Gamma)=\left\{\alpha \in \mathbb{R}^{N} \mid \sum_{i \in S} \alpha_{i}+\epsilon^{\min } \geq v^{\Gamma}(S) \text { for all } S \subset N, \text { and } \sum_{i \in N} \alpha_{i}=v^{\Gamma}(N)\right\}
$$

where

$$
\epsilon^{\min }=\min \left\{\epsilon \in \mathbb{R} \mid \sum_{i \in S} \alpha_{i}+\epsilon \geq v^{\Gamma}(S) \text { for all } S \subset N, \sum_{i \in N} \alpha_{i}=v^{\Gamma}(N)\right\} .
$$

Considering the definition of $\epsilon$-core, one can always find values of $\epsilon$ such that the $\epsilon$-core of corresponding CTLD situation is non-empty. Consequently, the leastcore, unlike the core, satisfies the NE property. However, the least-core does not necessarily results in a unique allocation (see Example 7 below) so it does not satisfy the UQ property. Since the least-core solely draws upon the CTLD game, it follows from Lemma 6 that it satisfies the IID property. It is straightforward to verify that in zero-normalized two-player games, the least-core contains only the allocation which divides the savings equally between the players which imply that it does not satisfy the RC property. Note that the definition of least-instability implies that the set of $\epsilon^{*}$-stable allocations for a CTLD situation always contains the corresponding $\epsilon^{\mathrm{min}}$ stable allocations. This is due to the fact that $\epsilon^{\mathrm{min}}$ can take negative values while $\epsilon^{*}$ is always non-negative. Therefore, when the core of a CTLD situation is not empty, the least-unstable allocations are within the core and when the latter is empty, the set of least-unstable allocations coincide with the least-core. Thus least-core satisfies the LU property.

The nucleolus (Schmeidler 1969) is another well-studied solution for cooperative games. As in the case of the core, the nucleolus in CTLD solutions can be defined as a mapping that assigns to every situation $\Gamma$ the nucleolus of its corresponding game. For a CTLD situation $\Gamma \in \mathcal{T}$ with player set $N$ and an allocation $\alpha$, define the vector of excesses $\theta(\alpha)$ as a vector in $\mathbb{R}^{2^{N}}$ whose components are the numbers $v^{\Gamma}(S)-\sum_{i \in S} \alpha_{i}$ arranged non-increasingly. For two vectors $x, x^{\prime} \in \mathbb{R}^{m}$, the lexicographical order $x \leq_{\text {lex }} x^{\prime}$ implies that either $x=x^{\prime}$, or there is $1 \leq t \leq m$ such that $x_{i}=x_{i}^{\prime}$ for $1 \leq j<t$ and $x_{t}<x_{t}^{\prime}$. Define the imputation set $M(\Gamma)=\left\{\alpha \mid \sum_{i \in N} \alpha_{i}=\right.$ $v^{\Gamma}(N)$ and $\alpha_{i} \geq 0$ for all $\left.i \in N\right\}$. The nucleolus of a CTLD situation $\Gamma$, i.e., $A^{\eta}(\Gamma)$, is the set of imputations whose associated vectors of excesses are lexicographically minimal:

$$
A^{\eta}(\Gamma)=\left\{\alpha \mid \theta(\alpha) \leq_{\text {lex }} \theta\left(\alpha^{\prime}\right) \text { for all } \alpha, \alpha^{\prime} \in M(\Gamma)\right\} .
$$

The nucleolus selects the allocations which lexicographically minimize the vector of objections for all coalitions of players where the objection is defined as the difference between the savings obtainable by that coalition and the given allocation. For every cooperative game, the nucleolus always exists, is unique, and is contained in the leastcore (Schmeidler 1969). Therefore, the nucleolus of CTLD situations satisfies the NE, 


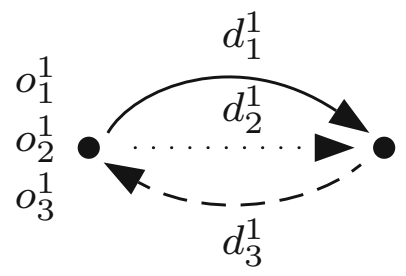

Fig. 6 A CTLD situation with three players

UQ, and LU properties. Since the nucleolus solely draws upon the CTLD game, it follows from Lemma 6 that it satisfies the IID property. However, it does not satisfy the RC property as in two-player situations it always results in the allocation that divides the savings equally between the two players (Aumann and Maschler 1985).

The Shapley value (Shapley 1953b) is a classic solution in cooperative game theory. The Shapley value of a game is a single-valued solution that allocates the savings to players based on their average contributions to all coalitions. By extending the notion of Shapley value to CTLD situations, we obtain the solution $A^{\Phi}$ wherein for situation $\Gamma$ with player set $N$ we have $A^{\Phi}(\Gamma)=\left\{\alpha^{\Phi}(\Gamma)\right\}$, that is for all $i \in N$ :

$$
\alpha_{i}^{\Phi}(\Gamma)=\sum_{S \subseteq N \backslash i} \frac{|S| !(n-|S|-1) !}{n !}\left[v^{\Gamma}(S \cup i)-v^{\Gamma}(S)\right] .
$$

For every CTLD situation, the corresponding Shapley value always exists and is unique. Since the Shapley value solely draws upon the CTLD game, it follows from Lemma 6 that it satisfies the IID property. Moreover, it always divides the savings equally in two-player situations (Aumann and Maschler 1985) thus it does not satisfy the RC property. The following example shows that the Shapley value does not satisfy the least-instability property.

Example 6 Consider the CTLD situation $\Gamma$ depicted in Fig. 6 . There are three players $N=\{1,2,3\}$ each having a depot at the same location and a delivery requirement. The distance between the pickup and delivery locations for all delivery requirements is one. The stand-alone cost for all players is 2. Although cooperation between players 1 and 2 does not create any savings, either of them can cooperate with player 3 to generate 2 units of savings. This results in $v^{\Gamma}(S)=0$ when $|S|=1, v^{\Gamma}(\{1,2\})=0$, $v^{\Gamma}(\{1,3\})=v^{\Gamma}(\{2,3\})=2$, and $v^{\Gamma}(N)=2$. The allocation $\alpha=\left(\alpha_{1}, \alpha_{2}, \alpha_{3}\right)=$ $(0,0,2)$ is the unique stable allocation. The Shapley value of the situation is $\alpha^{\Phi}(\Gamma)=$ $(1 / 3,1 / 3,4 / 3)$. Thus, the Shapley value is not least-unstable.

Weighted Shapley values (Shapley 1953a) extend the Shapley value by allowing unequal allocations of savings in two player situations based on exogenously given weights. Note that the Shapley value by itself is a weighted Shapley value where all players have equal weights. The exogenous weights reflect the different bargaining powers of the players which justifies discrimination among their allocations irrespective of the characteristics of the situation. In CTLD situations encountered by the authors, no player has ex ante a higher bargaining power over the others. 

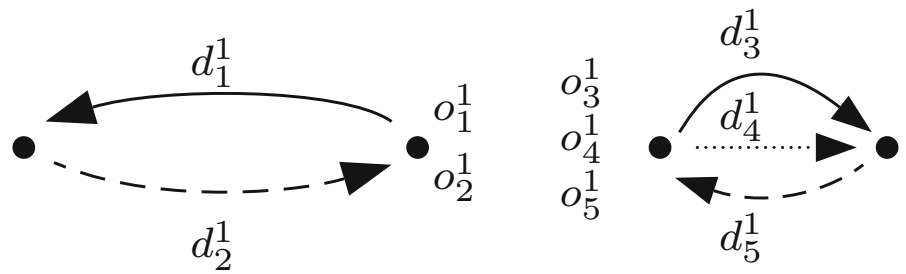

Fig. 7 A CTLD situation with five players

The alternative cost avoided (ACA) method introduced by Tijs and Driessen (1986) is a solution which draws upon the stand-alone costs of the players. We can adopt the ACA as a solution for CTLD situations. Let $\Gamma \in \mathcal{T}$ be a CTLD situation with player set $N$. Define $m_{i}=c^{*}\left(O_{N}, D_{N}\right)-c^{*}\left(O_{N \backslash i}, D_{N \backslash i}\right)$. The allocation of savings ${ }^{2}$ obtained by solution $A^{A C A}$ in situation $\Gamma$ is $A^{A C A}(\Gamma)=\left\{\alpha^{A C A}(\Gamma)\right\}$ where for all $i \in N$ :

$$
\alpha_{i}^{A C A}(\Gamma)=\left[c^{*}\left(O_{i}, D_{i}\right)-m_{i}\right]\left[1-\frac{\sum_{j \in N} m_{j}-c^{*}\left(O_{N}, D_{N}\right)}{\sum_{j \in N}\left(m_{j}-c^{*}\left(O_{j}, D_{j}\right)\right)}\right]
$$

This solution does not satisfy the NE property: in the situation depicted in Fig. 5 (Example 5) we have $m_{i}-c^{*}\left(O_{i}, D_{i}\right)=0$ for all $i \in N$ so $A^{A C A}(\Gamma)$ is not well defined for the situation in this example due to division by zero. Upon existence, $A^{A C A}$ obtains a single allocation. Definition of alternate cost avoided implies that the cost of irrelevant deliveries of a player $i, i \in N$, is additive both in $c^{*}\left(O_{i}, D_{i}\right)$ and $m_{i}$ so it cancels out in $c^{*}\left(O_{i}, D_{i}\right)-m_{i}$. Therefore, the exclusion of irrelevant deliveries does not affect $A^{A C A}$ and consequently it satisfies the IID property. It is straightforward to verify that in situations with two players only, the ACA always results in equal division of savings. Thus, it does not satisfy the RC property. Finally, the next example shows that the ACA does not satisfy the LU property.

Example 7 Consider the CTLD situation in Fig. 7 with $N=\{1,2,3,4,5\}$. Players 3 , 4 , and 5 each have a depot at the same location and a delivery with equal stand-alone costs of 2 (the distance between the two points at right is 1). Players 1 and 2 each also have a depot at the same location with a single delivery whose stand-alone costs is 4 (the distance between the two points at left is 2). The deliveries and depots in the left and right sides of the figure are distant enough so that no optimal trip can be constructed by combining the corresponding components. The stand-alone costs are $c^{*}\left(O_{1}, D_{1}\right)=$ $c^{*}\left(O_{2}, D_{2}\right)=4$ and $c^{*}\left(O_{3}, D_{3}\right)=c^{*}\left(O_{4}, D_{4}\right)=c^{*}\left(O_{5}, D_{5}\right)=2$. The saving obtained by coalition $\{1,2\}$ is 4 , the saving obtained by coalitions $\{3,4\},\{3,5\},\{4,5\}$, and $\{3,4,5\}$ are $0,2,2$, and 2 , respectively. The savings obtained by the grand coalition is 6 . The core of the associated game is non-empty and can be completely characterized by the allocations $\alpha=\left(\alpha_{1}, \alpha_{2}, \alpha_{3}, \alpha_{4}, \alpha_{5}\right)=(\theta, 4-\theta, 0,0,2)$ with $0 \leq \theta \leq 4$. With

\footnotetext{
2 Note that ACA is originally defined over cooperative cost games. We have modified the original ACA formula to describe corresponding allocations of savings rather than costs.
} 


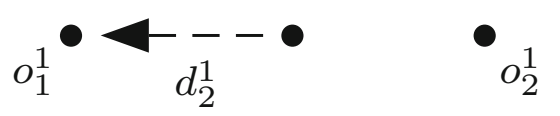

Fig. 8 A CTLD where $i$ has zero stand-alone cost

respect to ACA, we have $m_{1}=m_{2}=m_{5}=0$ and $m_{3}=m_{4}=2$. Consequently, we get $\alpha^{A C A}=(2.4,2.4,0,0,1.2)$. So $\alpha^{A C A}$ is not the least-unstable allocation.

The proportional solution introduced by Ortmann (2000) incorporates proportionality as a measure of fairness in division of savings. To provide the formula for this solution in CTLD situations, consider the situation $\Gamma=\left(N, V, w,\left(D_{i}\right)_{i \in N},\left(O_{i}\right)_{i \in N}, L\right)$ and accordingly define the restricted situation $\Gamma^{S}=\left(S, V, w,\left(D_{i}\right)_{i \in S},\left(O_{i}\right)_{i \in S}\right.$, $\left.L\left(O_{S}, D_{S}\right)\right)$ as the situation obtained by ignoring the players not in $S$. Note that $\Gamma^{N}=\Gamma$. The proportional solution a la Ortmann for CTLD situations is defined via

$$
A^{\psi}(\Gamma)=\left\{\left(c^{*}\left(O_{i}, D_{i}\right)-\psi_{i}\left(\Gamma^{N}\right)\right)_{i \in N}\right\}
$$

where for all $i \in N$ and $S \subseteq N, \psi_{i}\left(\Gamma^{S}\right)$ is obtained recursively from ${ }^{3}$

$$
\psi_{i}\left(\Gamma^{S}\right)=c^{*}\left(O_{S}, D_{S}\right)\left[1+\sum_{j \in S \backslash i} \frac{\psi_{j}\left(\Gamma^{S \backslash i}\right)}{\psi_{i}\left(\Gamma^{S \backslash j}\right)}\right]^{-1}
$$

with $\psi_{i}\left(\Gamma^{i}\right)=c^{*}\left(O_{i}, D_{i}\right)$. With the above definition, this solution only exists in situations where for every $i \in N$ it holds that $c^{*}\left(O_{i}, D_{i}\right) \neq 0$. This is not always the case in CTLD situations as shown in the next example.

Example 8 Consider the CTLD situation depicted in Fig. 8 with $N=\{1,2\}$. Player 1 has a depot but no deliveries and player 2 has a depot and a delivery which is close to player 1's depot. The distance between the adjacent points is 1 . Through cooperation the players can obtain two units of savings, i.e., $v^{\Gamma}(N)=2$, we have $c^{*}\left(O_{1}, D_{1}\right)=0$.

The example above shows that the proportional solution does not satisfy the NE property. Upon existence, the proportional solution provides a single allocation (Ortmann 2000). In CTLD situations with two players, the proportional solution divides savings in proportion to stand-alone costs of the players. As the result, this solution satisfies the RC property. However, considering the entire stand-alone cost players hinders this solution from satisfying the IID property. Looking back at Example 6, observe that the proportional solution $A^{\psi}$ obtains the allocation $\alpha^{\psi}=(2 / 5,2 / 5,6 / 5)$. Given that the only stable allocation in this situation is $\alpha=(0,0,2)$, we conclude that this solution does not satisfy the LU property.

\footnotetext{
${ }^{3}$ Note that the original solution is extended to incorporate proportionality with regard to costs, instead of savings.
} 
The equal profit-sharing method proposed by Frisk et al. (2010) addresses the concerns over stability as well as competitiveness in transportation situations. The EPM solution chooses allocations in the core, or in the least-core when the core is empty, which minimize the maximum difference between all pairwise ratios of allocation to stand-alone cost. In CTLD situations, the EPM solution is defined as $A^{\mathrm{EPM}}$ where for a situation $\Gamma$ :

$$
\begin{aligned}
& A^{\mathrm{EPM}}(\Gamma)=\underset{\alpha}{\arg \min f} \\
& \text { s.t. } \frac{\alpha_{i}}{c^{*}\left(O_{i}, D_{i}\right)}-\frac{\alpha_{j}}{c^{*}\left(O_{j}, D_{j}\right)} \leq f \quad \forall i, j \in N \\
& \sum_{i \in S} \alpha_{i}+\epsilon^{\min } \geq v^{\Gamma}(S) \quad \forall S \subset N \\
& \sum_{i \in N} \alpha_{i}=v^{\Gamma}(N)
\end{aligned}
$$

with $\epsilon^{\text {min }}$ being defined in (29). In CTLD situations with two players, the EPM allocates savings proportional to stand-alone costs of the players. Thus, $A^{\text {EPM }}$ satisfies the RC property. However, this solution does not satisfy the IID property as it considers the entire stand-alone costs of the players. Moreover, EPM is not defined for the case where the stand-alone cost of a player is zero (as in Fig. 8), thus it does not satisfy the NE property either. We return to Example 7 to show that EPM can produce more than a single allocation in some situations. With regard to later example, first note that $\epsilon^{\mathrm{min}}=$ 0 . Now consider the family of allocations $\left(\alpha_{1}, \alpha_{2}, \alpha_{3}, \alpha_{4}, \alpha_{5}\right)=(\theta, 4-\theta, 0,0,2)$ with $0 \leq \theta \leq 4$. Note that the largest difference between pairwise ratios of allocation to stand-alone cost in this family is 1 . In fact, all allocations in this family are optimal solutions to the program defined by (30)-(33). We conclude that $A^{\text {EPM }}$ does not satisfy the UQ property.

\section{Final remarks and conclusions}

In this paper, we proposed a solution for CTLD situations. The proposed solution satisfies a series of properties which reflect the requirements for fairness and/or competitiveness in these situations. The solution always exists, gives a unique allocation, and is situated within the core of the cooperative game associated with the situationor, if the latter is empty, the solution is within the least-core of the associated game (Theorem 2). The proposed solution is insensitive to the exclusion of deliveries which could not play any role in cooperation and satisfies a minimal requirement for competitiveness of allocations (Theorem 3).

Our solution for CTLD situations incorporates the notion of competitiveness in the sense of proportionality to competitive positions. To do so, the solution draws upon the average cost of fulfillment for MEDSs of players to define a measure for competitive positions of logistics providers. The minimal essential deliveries are subsets of delivery requirements of players which are necessary and sufficient in creating their contributions to the grand coalition and have the minimum stand-alone costs (Defi- 
nition 4). The preservation of competitive positions implies that the ratio of average cost of fulfillments of the players remain the same before and after cooperation.

To address fairness of the solution, we seek allocations that minimize the dissatisfaction of sub-coalitions. To do so we restrict our attention to the set of least-unstable allocations, i.e., the smallest non-empty $\epsilon$-core of the corresponding cooperative cost game $(\epsilon \geq 0)$. The non-negativity of $\epsilon$ reflects the fact that in CTLD situations, companies must invest to be able to cooperate. The investment includes establishing standardized information systems, and perhaps employing a transactional intermediary to ensure privacy of information which is critical in the competitive environment of logistics sector. Thus, least-unstable allocations render our allocations fair in a coalitional sense. Note that choosing among $\epsilon$-stable allocations with $\epsilon<0$ may yield an allocation further away from the proportional solution $A^{P}$ without meaningfully improving the stability of the allocation.

Ideally, our solution yields the allocation which equalizes the ratios of average costs of deliveries before and after cooperation for all players, i.e., the proportional allocation. However, with more than two players the proportional allocation need not be situated within the core of the associated cooperative cost game (or within the least-core when the core is empty). To avoid impossibility results, we defined the competitive requirements of the solution in a restricted manner and in terms of two-player situations. In the last step, our solution selects the point in the core of the associated cooperative game (or the least-core when the latter is empty) that has the shortest distance from the proportional allocation.

The solution is proposed with special attention to implementability considerations. The preliminary allocation introduced in this paper, i.e., $A^{P}$, which draws upon MEDSs of players, can be calculated by comparing the individual optimal delivery plans of the logistics providers in stand-alone mode versus those in the grand coalition. This is due to the fact that the essential delivery sets are detectable from the latter comparison (as implied by Lemma 5). In practical instances where the multiplicity of optimal delivery plans are improbable, the essential delivery sets of the players can be obtained by detecting the deliveries whose fulfillment in the grand coalition involves other players, that is, the trips including those deliveries are either initiated from another player's depot or they include deliveries of others. When the essential delivery sets are multiple, finding the minimal sets among them requires comparison among their stand-alone delivery costs.

To the best of our knowledge, this paper is the first to formally incorporate an endogenous measure of competitiveness in logistics markets. This is done by considering the lowest possible price that a logistics provider is able to charge for a unit distance of its delivery services within a specified scope without incurring loss. Such a measure reflects the internal efficiency of the logistics providers' operations. Consequently, our solution takes advantage of information contained in a situation in addition to the savings generated in all possible coalitions to calculate the allocation.

We argue that in cooperative operations management, investigating the desirable properties of solutions and their formal definition ex ante obtains more meaningful results than using generic solutions. Without having a solid ground for comparing among different solutions, one cannot objectively evaluate the performance of various available solutions. The definition of desirable properties in CTLD situations in this 
paper allows us to conduct such comparison. The results clarify the advantages of our proposed solution over some of the existing solutions in the literature which are more suited to be adopted in these situations.

As a practical advantage of our proposed solution, it can be extended to handle more realistic CTLD situations. The restrictions for delivery time windows, requirements for trip lengths, availability of personnel and shifts can all be easily included in the basic model which obtains costs. Despite possible challenges in solving the optimization problem, as long as one can identify the best joint plans for fulfilling the delivery requirements in the grand coalition, our approach in obtaining competitive allocations remains valid for cooperating logistics players.

Open Access This article is distributed under the terms of the Creative Commons Attribution License which permits any use, distribution, and reproduction in any medium, provided the original author(s) and the source are credited.

\section{References}

Audy J, D'Amours S, Rousseau L (2010) Cost allocation in the establishment of a collaborative transportation agreement — an application in the furniture industry. J Oper Res Soc 62(6):960-970

Aumann RJ, Maschler M (1985) Game theoretic analysis of a bankruptcy problem from the Talmud. J Econ Theory 36(2):195-213

Caprara A, Letchford A (2010) New techniques for cost sharing in combinatorial optimization games. Math Program 124(1-2):93-118

Cruijssen F, Cools M, Dullaert W (2007) Horizontal cooperation in logistics: opportunities and impediments. Transp Res Part E 43(2):129-142

Curiel I (2008)Cooperative combinatorial games. In: Pareto optimality, game theory and equilibria. Springer, New York, pp 131-157

Dai B, Chen H (2012) Profit allocation mechanisms for carrier collaboration in pickup and delivery service. Comput Ind Eng 62(2):633-643

Davidson K, Donsig A (2010) Real analysis and applications: theory in practice. Springer, New York

Drechsel J, Kimms A (2010) Computing core allocations in cooperative games with an application to cooperative procurement. Int J Prod Econ 128(1):310-321

Engevall S, Göthe-Lundgren M, Värbrand P (2004) The heterogeneous vehicle-routing game. Transp Sci 38(1):71-85

Eurostat (2012) Annual road freight transport vehicle movements, loaded and empty, by reporting country. http://appsso.eurostat.ec.europa.eu/nui/show.do?dataset=road_go_ta_vm\&lang=en Accessed 2 March 2015

Frisk M, Göthe-Lundgren M, Jörnsten K, Rönnqvist M (2010) Cost allocation in collaborative forest transportation. Eur J Oper Res 205(2):448-458

Fujishige S, Isotani S (2011) A submodular function minimization algorithm based on the minimum-norm base. Pac J Optim 7(1):3-17

Gillies D (1959) Solutions to general non-zero-sum games. Contrib Theory Games 4:47-85

Göthe-Lundgren M, Jörnsten K, Värbrand P (1996) On the nucleolus of the basic vehicle routing game. Math Program 72(1):83-100

Granot D, Hamers H, Tijs S (1999) On some balanced, totally balanced and submodular delivery games. Math Program 86(2):355-366

Hamers H (1997) On the concavity of delivery games. Eur J Oper Res 99(2):445-458

Hamers H, Borm P, van de Leensel R, Tijs S (1999) Cost allocation in the Chinese postman problem. Eur J Oper Res 118(1):153-163

Hezarkhani B, Slikker M, Van Woensel T (2014) On characterization of the core of lane covering games via dual solutions. Oper Res Lett 42(8):505-508 
Krajewska M, Kopfer H, Laporte G, Ropke S, Zaccour G (2007) Horizontal cooperation among freight carriers: request allocation and profit sharing. J Oper Res Soc 59(11):1483-1491

Liu P, Wu Y, Xu N (2010) Allocating collaborative profit in less-than-truckload carrier alliance. J Serv Sci Manag 3(1):143-149

Lozano S, Moreno P, Adenso-Díaz B, Algaba E (2013) Cooperative game theory approach to allocating benefits of horizontal cooperation. Eur J Oper Res 229(2):444-452

Maschler M, Peleg B, Shapley L (1979) Geometric properties of the kernel, nucleolus, and related solution concepts. Math Oper Res 4(4):303-338

Ortmann M (2000) The proportional value for positive cooperative games. Math Methods Oper Res 51(2):235-248

Özener O, Ergun Ö (2008) Allocating costs in a collaborative transportation procurement network. Transp Sci 42(2):146-165

Platz T, Hamers H (2013) On games arising from multi-depot Chinese postman problems. Tech. rep., Tilburg University, Center for Economic Research

Schmeidler D (1969) The nucleolus of a characteristic function game. SIAM J Appl Math 17(6):1163-1170

Shapley L (1953a) Additive and non-additive set functions. PhD thesis, Princeton University, Princeton

Shapley L (1953b) A value for $n$-person games. In: Contributions to the theory of games, vol II. Princeton University Press, Princeton, pp 307-317

Shapley LS, Shubik M (1966) Quasi-cores in a monetary economy with nonconvex preferences. Econ J Econ Soc 34:805-827

Tijs S, Driessen T (1986) Game theory and cost allocation problems. Manag Sci 32(8):1015-1028

Vanovermeire C, Vercruysse D, Sörensen K (2013) Analysis of different cost allocation methods in a collaborative transport setting. Working papers, University of Antwerp, Faculty of Applied Economics

Wolfe P (1976) Finding the nearest point in a polytope. Math Program 11(1):128-149 